\title{
Building SO(10) models from F-theory
}

\author{
I. Antoniadis ${ }^{a, 1}$ and G.K. Leontaris ${ }^{b}$ \\ a Department of Physics, CERN Theory Division, \\ CH-1211, Geneva 23, Switzerland \\ ${ }^{b}$ Physics Department, Theory Division, Ioannina University, \\ GR-45110 Ioannina, Greece \\ E-mail: Ignatios.Antoniadis@cern.ch, leonta@uoi.gr
}

AbSTRACT: We revisit local F-theory SO(10) and SU(5) GUTs and analyze their properties within the framework of the maximal underlying $E_{8}$ symmetry in the elliptic fibration. We consider the symmetry enhancements along the intersections of seven-branes with the GUT surface and study in detail the embedding of the abelian factors undergoing monodromies in the covering gauge groups. We combine flux data from the successive breaking of $\mathrm{SO}(10)$ to $\mathrm{SU}(5)$ gauge symmetry and subsequently to the Standard Model one, and further constrain the parameters determining the models' particle spectra. In order to eliminate dangerous baryon number violating operators we propose ways to construct matter parity like symmetries from intrinsic geometric origin. We study implementations of the resulting constrained scenario in specific examples obtained for a variety of monodromies.

KEYWORDS: Strings and branes phenomenology

ARXiv EPRINT: 1205.6930

\footnotetext{
${ }^{1}$ On leave from CPHT (UMR CNRS 7644) Ecole Polytechnique, F-91128 Palaiseau, France.
} 


\section{Contents}

1 Introduction 1

2 F-theory model building $\quad 2$

$2.1 \mathcal{Z}_{2}$ monodromy 4

2.1.1 Flux restrictions and multiplicities 4

$\begin{array}{lll}2.1 .2 & \mathrm{SU}(5) \text { spectrum } & 6\end{array}$

$2.2 \mathcal{Z}_{2} \times \mathcal{Z}_{2}$ monodromy $\quad 7$

2.2.1 $\mathrm{SU}(5)$ spectrum $\quad 8$

$\begin{array}{lll}2.3 & \mathcal{Z}_{3} \text { monodromy } & 8\end{array}$

$\begin{array}{lll}2.3 .1 & \mathrm{SU}(5) \text { spectrum } & 9\end{array}$

$\begin{array}{llr}3 & \text { Low energy effective models } & 10\end{array}$

$\begin{array}{lll}3.1 & \mathcal{Z}_{2} \text { models } & 12\end{array}$

$\begin{array}{lll}3.1 .1 & \text { Model } A & 14\end{array}$

$\begin{array}{lll}3.1 .2 & \text { Model } B & 16\end{array}$

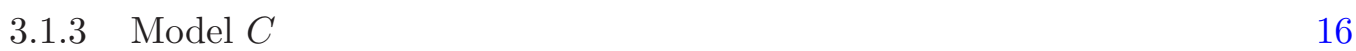

$\begin{array}{lll}3.2 & \mathcal{Z}_{2} \times \mathcal{Z}_{2} \text { models } & 18\end{array}$

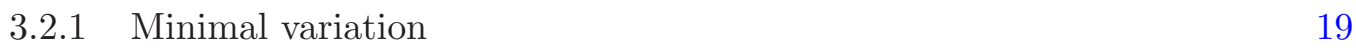

$\begin{array}{lll}3.3 & \mathcal{Z}_{3} \text { models } & 21\end{array}$

$\begin{array}{lll}3.3 .1 & \text { First example } & 21\end{array}$

$\begin{array}{lll}3.3 .2 & \text { Second example } & 23\end{array}$

4 Matter parity from geometry $\quad \mathbf{2 4}$

4.1 A $Z_{2}$ parity for the $\mathcal{Z}_{2} \times \mathcal{Z}_{2}$ monodromy $\quad 25$

4.2 The case of $\mathcal{Z}_{3}$ models $\quad 26$

$\begin{array}{lll}4.3 & \text { Matter parity for the } \mathcal{Z}_{2} \text { model } C & 27\end{array}$

4.4 A $Z_{2}$ matter parity for a model with $2+3$ spectral cover split $\quad 27$

$\begin{array}{llr}5 & \text { Conclusions } & 29\end{array}$

A F-SO(10) gauge symmetry and enhancements $\quad 30$

A.1 Review of the Weierstrass form and some related material 31

$\begin{array}{lll}\text { A.2 } & \mathrm{SO}(10) & 32\end{array}$

A.2.1 Symmetry enhancement 33

$\begin{array}{lll}\text { A.2.2 Extraneous matter } & 35\end{array}$

$\begin{array}{lll}\text { A.3 Polynomial equations for the matter curves } & 36\end{array}$

$\begin{array}{ll}\text { B Monodromies } & 38\end{array}$

$\begin{array}{lll}\text { B.1 Symmetry enhancements and } \mathcal{Z}_{2} \text { monodromy } & 38\end{array}$

B.1.1 Enhancements along intersecting matter curves 40

B.1.2 $\mathrm{SU}(5)$ and spectral cover splitting for the $\mathcal{Z}_{2}$ case 43 
B.2 $\mathcal{Z}_{2} \times \mathcal{Z}_{2}$ spectral cover factorization

B.2.1 The SU(5) case $\quad 45$

$\begin{array}{lll}\text { B.3 } & \mathcal{Z}_{3} \text { factorization } & 46\end{array}$

$\begin{array}{lll}\text { B.3.1 } & \mathrm{SU}(5) \text { with } \mathcal{Z}_{3} \text { monodromy } & 47\end{array}$

\section{Introduction}

F-theory [1] provides an interesting 'geometrical' reformulation of type IIB superstring theory. It is envisaged that this new approach will open new possibilities and prove particularly useful to string model building. Indeed, during the last few years there is ample evidence that old successful GUTs including the minimal $\mathrm{SU}(5)$, the $\mathrm{SO}(10)$ model etc., are naturally realized in F-theory compactifications [2]-[11]. The rather interesting fact in F-theory constructions is that because they are defined on a compact elliptically fibered Calabi-Yau complex four dimensional Manifold the exceptional groups $E_{6}, E_{7}, E_{8}$, can be naturally incorporated into the theory too $[2,3,5,12]$. Although exceptional gauge symmetries when realized in the context of four-dimensional grand unified theories suffer from several drawbacks, in the case of F-theory models they appear to be more promising as new possibilities arise for the symmetry breaking mechanisms and the derivation of the desired massless spectrum. Indeed, an alternative possibility is to turn on appropriate $\mathrm{U}(1)$ fluxes on the worldvolume of the 7-brane. Applying this mechanism to SU(5) for example it is possible to obtain zero modes for the Higgs doublets without having dangerous light triplets since they belong to different Dolbeault cohomology groups $[2,3,5]$. Of course the ultimate goal to build a low energy effective theory with the required spectrum and acceptable quark and lepton mass matrices from first principles is a formidable task. Precious tools towards this goal are provided by the twisted eight-dimensional Yang-Mills theory and the particular geometry of the 'internal space' which determines the wavefunctions' profiles of the various elementary particles. This information as well as the successful implementation of the novel GUT breaking mechanism with fluxes on the doublet-triplet problem are convincing enough to motivate us to delve into the F-theory vacua searching for the ultimate model.

A particularly useful ingredient in building realistic F-theory models, is the notion of monodromy [12-14]. In F-theory configurations involving intersecting seven branes, matter fields appear to localize on the intersections of two such branes, while the Yukawa couplings occur when three of these branes intersect at a common point. Computations of the matter fields wavefunctions $[13,15,16]$, show that these fields are localized at a very tiny region of this intersection. Monodromy ensures the appearance of tree-level mass for the top quark and rank-one tree-level fermion mass matrices [15]-[24], while it plays a decisive role on the final shape of effective models [24]-[32]. The strength of the corresponding Yukawa couplings are given by integrals of the overlapping wavefunctions picked at the point of the triple intersection $[13,15,16,22,25,27,33,34] .{ }^{1}$

\footnotetext{
${ }^{1}$ For similar Yukawa couplings calculations in different context see also [35-39].
} 
In the present work we use spectral cover techniques and attempt to thoroughly analyze the $\mathrm{SO}(10)$ as well as the (after the symmetry breaking) emerging SU(5) GUT, with respect to a variety of choices for the monodromy. In our study we assume that there exists a single point of $E_{8}$ enhancement in the internal geometry [12] with matter descending from the adjoint of $E_{8}$. From the breaking patterns $E_{8} \supset \mathrm{SO}(10) \times \mathrm{SU}(4)_{\perp}$ and $E_{8} \supset$ $\mathrm{SU}(5) \times \mathrm{SU}(5)_{\perp}$ it turns out that the spectral covers possess $\mathrm{SU}(4)$ and $\mathrm{SU}(5)$ symmetries, respectively. Thus, extending previous works, we classify $\mathrm{SO}(10)$ models according to the non-trivial monodromy groups which are subgroups of the Weyl group $W\left(\mathrm{SU}(4)_{\perp}\right)=S_{4}$. Considering in particular the spectral cover symmetry breaking to the Cartan subalgebra $\mathrm{SU}(4)_{\perp} \rightarrow \mathrm{U}(1)^{3}$, we study the quotient theory after imposing identifications of these abelian factors under the subgroups $\mathcal{Z}_{2}, \mathcal{Z}_{3}$, and the Klein four-group $\mathcal{Z}_{2} \times \mathcal{Z}_{2}$. Further, we assume successive breaking of the $\mathrm{SO}(10)$ symmetry to $\mathrm{SO}(10) \rightarrow \mathrm{SU}(5) \times \mathrm{U}(1)_{X}$ through a U $(1)_{X}$-flux and the $\mathrm{SU}(5)$ to the Standard Model (SM) by a $\mathrm{U}(1)_{Y}$ (hypercharge) flux. A novel feature of our analysis is the fact that we impose combined constraints from both $\mathrm{U}(1)_{X, Y}$ fluxes to further restrict the flux parameters of the model.

Using factorization techniques of the spectral cover equations we derive the properties and in particular the homology classes of the matter curves which constitute an essential tool towards the computation of flux restrictions and the chiral matter spectrum of the models. Because monodromies play a particular role on various aspects - including computations of the Yukawa couplings - we pay particular attention in the way U(1) symmetries are involved in monodromies. Indeed, matter curves are characterized by the $\mathrm{U}(1)$ symmetries and as a result, the monodromy action would identify some of them. At the next stage of the present work, we combine the obtained data from our analysis and investigate their implications on the unspecified parameters and superpotential couplings of viable effective low energy models. Among other things, we discuss doublet triplet splitting, proton decay and fermion masses. To eliminate dimension-four baryon number violating operators we appeal to the geometric properties of the internal manifold and suggest ways to construct $Z_{2}$ discrete symmetries associating them to a possible matter parity. We present examples where such constructions lead to viable effective low energy models and compare them with the existing ones in the recent literature.

More precisely in section 2 we analyze in detail the various monodromies of $\mathrm{SO}(10)$ and SU(5) models and the general geometric and flux constraints imposed on the matter spectrum. In section 3 we elaborate several effective models and in section 4 we attempt to construct matter parity consistent with the intrinsic geometry. We summarize the results and present our conclusions in section 5. To make this paper self-contained, in the appendix we review the necessary F-theory tools used in our work, including the Weierstrass equation and Tate's forms. Moreover we investigate the gauge symmetry enhancements along intersecting matter curves, and present details of our calculations.

\section{F-theory model building}

F-theory is defined on a background $R^{3,1} \times \mathcal{X}$ with $R^{3,1}$ the space-time and $\mathcal{X}$ an elliptically fibered Calabi-Yau complex fourfold over a complex three-fold base. In this work 
we will describe elliptic fibration through the well known Weierstass model given by the equation (A.1) of the appendix. According to the 'standard' interpretation in F-theory the gauge symmetry is associated to the singularities of the internal compact manifold. In this work we assume a geometric singularity of a two-complex dimension Kähler surface $S_{\mathrm{GUT}}$ identified with a group $G_{S}=\mathrm{SO}(10){ }^{2}$ The group structure of the spectral cover is the commutant of $G_{S}$ to $\mathcal{E}_{8}$

$$
\mathcal{E}_{8} \supset \mathrm{SO}(10) \times \mathrm{SU}(4)_{\perp} \rightarrow \mathrm{SO}(10) \times \mathrm{U}(1)^{3}
$$

where we assumed further breaking of $\mathrm{SU}(4) \rightarrow \mathrm{U}(1)^{3}$ by a non-zero adjoint Higgs vev. All matter is then found in the representations obtained from the decomposition of the $\mathcal{E}_{8}$-adjoint under the breaking pattern $(2.1)$

$$
248 \rightarrow(45,1)+\left(16,4_{\perp}\right)+\left(\overline{16}, \overline{4}_{\perp}\right)+\left(10,6_{\perp}\right)+\left(1,15_{\perp}\right)
$$

Each $\mathrm{SO}(10)$ representation $R$ resides on a matter curve $\Sigma_{R}$ which is distinguished by the specific charge it carries under the $\mathrm{U}(1)^{3}$ Cartan subalgebra, characterized by the $\mathrm{SU}(4)_{\perp}$ weights $t_{i}$

$$
\Sigma_{16}: \quad t_{i} ; \quad \Sigma_{10}: t_{i}+t_{j} ; \quad \Sigma_{1}: \pm\left(t_{i}-t_{j}\right), \quad i \neq j
$$

while in addition there are three singlets with zero weight.

Investigating the properties of semi-local models, we can extract useful information for the matter curves using Tate's Algorithm [42]. In particular for the SO(10) singularity the Weierstrass equation - associated to the elliptic fibration of the internal manifold takes the form

$$
y^{2}=x^{3}+b_{5} x y z+b_{4} x^{2} z+b_{3} y z^{2}+b_{2} x z^{3}+b_{0} z^{5}
$$

with $x, y$ being homogeneous coordinates of the torus fiber and $b_{k}$ are functions of the coordinates of the three-fold base. These are sections of $\left[b_{k}\right]=\eta-k t$, where $\eta=6 c_{1}-t$ with $c_{1},-t$ being the first Chern classes of the tangent and normal bundle to $S_{\mathrm{GUT}}$ respectively. The singularity enhancements where chiral matter is found are studied in detail in the appendix. Important properties of the local model are also 'encoded' in the spectral cover equation which for the $\mathrm{SU}(4)$ case reads

$$
\mathcal{C}_{4}=\sum_{k=1}^{4} b_{k} s^{4-k}=0
$$

with $s$ being an affine parameter. Furthermore, a detailed analysis summarized in the appendix shows that the 'locations' of the seven branes associated to the $\mathrm{SO}(10)$ nontrivial representations are determined from the equations

$$
16: b_{4}=0, \quad 10: \quad b_{3}^{2}=0
$$

The three additional U(1)'s in the breaking pattern of equation (2.1) are expected to put constraints on the superpotential couplings of the effective low energy model. In

\footnotetext{
${ }^{2}$ For previous studies of F-SO(10) models see for example [3, 28, 31, 40, 41].
} 


\begin{tabular}{|c|c|c|c|c|}
\hline Matter & $t_{i}$ charges & Section & Homology & $\mathrm{U}(1)_{X}$ \\
\hline $\mathbf{1 6}$ & $t_{1,2}$ & $a_{1}$ & $\eta-2 c_{1}-\chi$ & $M-P$ \\
$\mathbf{1 6}$ & $t_{3}$ & $a_{4}$ & $-c_{1}+\chi_{5}$ & $P_{5}$ \\
$\mathbf{1 6}$ & $t_{4}$ & $a_{6}$ & $-c_{1}+\chi_{7}$ & $P_{7}$ \\
$\mathbf{1 0}$ & $t_{1,2}+t_{3}$ & $\left(a_{1}-\lambda a_{4} a_{6}\right)$ & $\eta-2 c_{1}-\chi$ & $M-P$ \\
$\mathbf{1 0}$ & $t_{1,2}+t_{4}$ & $\left(a_{1}-\lambda a_{4} a_{6}\right)$ & $\eta-2 c_{1}-\chi$ & $M-P$ \\
$\mathbf{1 0}$ & $t_{1}+t_{2}$ & $\left(a_{5} a_{6}+a_{4} a_{7}\right)$ & $-c_{1}+\chi$ & $P$ \\
$\mathbf{1 0}$ & $t_{3}+t_{4}$ & $\left(a_{5} a_{6}+a_{4} a_{7}\right)$ & $-c_{1}+\chi$ & $P$ \\
\hline
\end{tabular}

Table 1. Properties of SO(10) representations in the $\mathcal{Z}_{2}$ monodromy.

particular the simultaneous existence of all three U(1) symmetries would not allow a treelevel Yukawa coupling for the top quark - ubiquitous in all viable fermion textures. This indicates the existence of possible monodromies among the U(1)'s allowing the emergence of a rank one fermion mass matrix structure. In the present case the possible monodromies are associated to $\mathcal{Z}_{2}, \mathcal{Z}_{3}$ and $\mathcal{Z}_{2} \times \mathcal{Z}_{2}$ discrete symmetries leading to the equivalent $\mathcal{C}_{2+1+1}$, $\mathcal{C}_{3+1}, \mathcal{C}_{2+2}$ factorizations of the spectral cover. In other words, these imply the identification of two weights $\left\{t_{1}, t_{2}\right\}$ or three of them $\left\{t_{1}, t_{2}, t_{3}\right\}$ or pairing them in two distinct sets $\left\{t_{1}, t_{2}\right\},\left\{t_{3}, t_{4}\right\}$ correspondingly. Each of these cases leads to a different class of low energy models which we examine in the subsequent sections.

\section{$2.1 \quad \mathcal{Z}_{2}$ monodromy}

In this case the factorization of the spectral cover equation is

$$
\mathcal{C}_{4}=\left(a_{1}+a_{2} s+a_{3} s^{2}\right)\left(a_{4}+a_{5} s\right)\left(a_{6}+a_{7} s\right)
$$

Comparing this to (2.5) we extract equations of the form $b_{k}=b_{k}\left(a_{i}\right)$ and use them to derive the relations for the homologies $\left[a_{i}\right]$ of the coefficients $a_{i}$. These are of the form

$$
\eta-k c_{1}=\left[a_{l}\right]+\left[a_{m}\right]+\left[a_{n}\right], \quad \text { with } \quad k+l+m+n=15
$$

where $l, m, n$ take the values $1,2, \ldots, 7$ and $k=0,1,2,3,4$. Note that we also need to solve the constraint $b_{1}\left(a_{i}\right)=0$ adopting a suitable Ansatz for some $a_{i}$ coefficients. The solution can be expressed in terms of two arbitrary parameters $\chi_{5}=\left[a_{5}\right], \chi_{7}=\left[a_{7}\right]$. We present these details the appendix.

The above can be used to determine the topological properties of matter curves. In particular, from the equation $b_{4}=a_{1} a_{4} a_{6}=0$ we deduce that the three $\mathbf{1 6}$ 's left after the monodromy action are determined by $a_{1}=0, a_{4}=0$ and $a_{6}=0$. Similarly, the equation $b_{3}^{2}\left(a_{i}\right)=0$ determines the properties of $\mathbf{1 0}$ 's. We collect all the results in table 1 where we introduce for convenience $\chi=\chi_{5}+\chi_{7}$.

\subsubsection{Flux restrictions and multiplicities}

To proceed further, we investigate the implications of the gauge symmetry breaking of $\mathrm{SO}(10)$ to $\mathrm{SU}(5) \times \mathrm{U}(1)_{X}$ by a $\mathrm{U}(1)_{X}$ flux. We introduce the following notation for the 
$\mathrm{U}(1)_{X}$ flux parameters

$$
\begin{aligned}
M & =\mathcal{F}_{1} \cdot\left(\eta-3 c_{1}\right) \\
P & =\mathcal{F}_{1} \cdot\left(\chi-c_{1}\right) \\
P_{n} & =\mathcal{F}_{1} \cdot\left(\chi_{n}-c_{1}\right), \quad n=5,7 \\
C & =-\mathcal{F}_{1} \cdot c_{1}
\end{aligned}
$$

This way we obtain the results of the last column of table 1 . We should mention that if we wish to protect the $\mathrm{U}(1)_{X}$ boson from receiving a Green-Schwartz (GS) mass we need to impose

$$
\mathcal{F}_{1} \cdot \eta=0, \quad \& \quad \mathcal{F}_{1} \cdot c_{1}=0
$$

which automatically imply $M=C=0$. In this case, the sum $P=P_{5}+P_{7}$ stands for the total flux permeating matter curves while one can observe form table 1 that the flux vanishes independently on the $\Sigma_{16}$ and $\Sigma_{10}$ matter curves.

Assuming that $M_{10}^{a}$ is the number of $\mathbf{1 0}_{t_{1}+t_{3}} \in \mathrm{SO}(10)$, after the $\mathrm{SO}(10)$ breaking we obtain the multiplicities

$$
\mathbf{1 0}_{t_{1}+t_{3},-t_{2}-t_{4}} \rightarrow \begin{cases}5_{-t_{2}-t_{4}} & M_{10}^{a}+(M-P)=M_{5_{2}} \\ \overline{5}_{t_{1}+t_{3}} & M_{10}^{a}-(M-P)=-M_{5_{1}}\end{cases}
$$

Similarly, if $M_{10}^{b}$ is the number of $\mathbf{1 0}_{t_{3}+t_{4}} \in \mathrm{SO}(10)$, we get

$$
\mathbf{1 0}_{t_{3}+t_{4},-t_{1}-t_{2}} \rightarrow \begin{cases}5_{-t_{1}-t_{2}} & M_{10}^{b}+P=M_{5_{u}} \\ \overline{5}_{t_{3}+t_{4}} & M_{10}^{b}-P=-M_{5_{4}}\end{cases}
$$

Now, let $M_{16}^{1}$ the multiplicity of $16_{t_{1}}$. Again from table 1 we have ${ }^{3}$

$$
\mathbf{1 6}_{t_{1,2}}=\left\{\begin{array}{lll}
10_{t_{1,2}} & M_{16}^{1} & =M_{10_{1}} \\
\overline{5}_{t_{1,2}+t_{5}} & M_{16}^{1}-(M-P)=-M_{5_{3}} \\
1_{t_{1,2}-t_{5}} & M_{16}^{1}+(M-P)=M_{S_{1}}
\end{array}\right.
$$

Continuing as above:

$$
\begin{array}{r}
\mathbf{1 6}_{t_{3}}= \begin{cases}10_{t_{3}} & M_{16}^{3}=M_{10_{2}} \\
\overline{5}_{t_{3}+t_{5}} & M_{16}^{3}-P_{5}=-M_{5_{h_{u}}} \\
1_{t_{3}-t_{5}} & M_{16}^{3}+P_{5}=M_{S_{35}}\end{cases} \\
\mathbf{1 6}_{t_{4}}= \begin{cases}10_{t_{4}} & M_{16}^{4}=M_{10_{3}} \\
\overline{5}_{t_{4}+t_{5}} & M_{16}^{4}-P_{7}=-M_{5_{6}} \\
1_{t_{4}-t_{5}} & M_{16}^{4}+P_{7}=M_{S_{45}}\end{cases}
\end{array}
$$

\footnotetext{
${ }^{3}$ Let us clarify that we interpret the multiplicities obtained from 16-decompositions as follows:

$$
\# \overline{5}_{t_{1,2}+t_{5}}-\# 5_{-t_{1,2}-t_{5}}=M_{16}^{1}-(M-P)
$$

and so on. This is subsequently identified with $-M_{5_{3}}$, so for the 5's of SU5 we will count: $M_{5_{i}}=\# 5-\# \overline{5}$. For later use we denote $M_{5_{1,2,3} \ldots}$ the multiplicities of $5^{\prime} s \in \mathrm{SU}(5)$. Numbering of $10_{j}, 5_{i}$ 's has been chosen to comply with the notation $M_{5_{i}} \ldots$ of refs $[21,22]$.
} 
where $M_{10_{i}}, M_{5_{j}}$ stand for the numbers of $10 \in \mathrm{SU}(5)$ and $5 \in \mathrm{SU}(5)$ representations (a negative value corresponds to the conjugate representation). $M_{S_{i j}}$ denote the multiplicities of the singlet fields. In fact, as for any other representation, this means that

$$
M_{i j}=\# 1_{t_{i}-t_{j}}-\# 1_{t_{j}-t_{i}}
$$

thus, if $M_{i j}>0$ then there is an excess of $M_{i j}$ singlets $1_{t_{i}-t_{j}}=\theta_{i j}$ and vice versa.

\subsection{2 $\mathrm{SU}(5)$ spectrum}

Next we consider the breaking of the $\mathrm{SU}(5)$ symmetry by the hypercharge $\left(\mathrm{U}(1)_{Y}\right)$ flux. The case of $\mathcal{Z}_{2}$ monodromy corresponds to the following splitting of the spectral cover equation

$$
b_{0} \prod_{i}\left(s-t_{i}\right)=\left(a_{1}+a_{2} s+a_{3} s^{2}\right)\left(a_{4}+a_{5} s\right)\left(a_{6}+a_{7} s\right)\left(a_{8}+a_{9} s\right)=\sum_{k=0}^{5} b_{k} s^{5-k}
$$

Following [21] we derive the relations of $b_{k}\left(a_{i}\right)$ by equating coefficients of the same powers in $s$, while we use a suitable Ansatz for $a_{i}$ 's to solve the constraint $b_{1}\left(a_{i}\right)=0$. The tenplets are found by studying the zeroth order of the above polynomial, which is $b_{5}=a_{1} a_{4} a_{6} a_{8}$. These are designated as

$$
10_{t_{1}}^{(1)}, 10_{t_{3}}^{(2)}, 10_{t_{4}}^{(3)}, 10_{t_{5}}^{(4)}
$$

while their homologies are identified as those of $a_{1}, a_{4}, a_{6}, a_{8}$ respectively. To determine the properties of the fiveplets we need the corresponding spectral cover equation. This is a 10-degree polynomial

$$
\mathcal{P}_{10}(s) \propto \sum_{n=1}^{10} c_{n} s^{10-n}=b_{0} \prod_{i, j}\left(s-t_{i}-t_{j}\right), \quad i<j, \quad i, j=1, \ldots, 5
$$

Using (2.13) we can convert the coefficients $c_{n}=c_{n}\left(t_{j}\right)$ to functions of $c_{n}\left(b_{j}\right)$. In particular we are interested for the value $\mathcal{P}_{10}(0)$ given by the coefficient $c_{10}$ which can be expressed in terms of $b_{k}$ according to

$$
c_{10}\left(b_{k}\right)=b_{3}^{2} b_{4}-b_{2} b_{3} b_{5}+b_{0} b_{5}^{2}=0
$$

Using the equations $b_{k}\left(a_{i}\right)$ and the Ansatz, we can split this equation into seven factors which correspond to the seven distinct fiveplets left after the $\mathcal{Z}_{2}$ monodromy action:

$$
\begin{aligned}
P_{5}= & \left(a_{4} a_{7} a_{9}+a_{5}\left(a_{7} a_{8}+a_{6} a_{9}\right)\right) \times\left(a_{1}-c a_{4}\left(a_{7} a_{8}+a_{6} a_{9}\right)\right) \times\left(a_{1}-c a_{6}\left(a_{5} a_{8}+a_{4} a_{9}\right)\right) \\
& \times\left(a_{1}-c\left(a_{5} a_{6}+a_{4} a_{7}\right) a_{8}\right) \times\left(a_{5} a_{6}+a_{4} a_{7}\right) \times\left(a_{5} a_{8}+a_{4} a_{9}\right)\left(a_{7} a_{8}+a_{6} a_{9}\right)
\end{aligned}
$$

These are assigned as $5^{(0)}, 5^{(1)}, 5^{(2)}, 5^{(3)}, 5^{(4)}, 5^{(5)}, 5^{(6)}$ respectively, while their homologies can be specified using those of $a_{i}$ given in table 25 of the appendix. Notice that three of the above factors correspond to three fiveplets of the same homology class $\left[a_{1}\right]=\eta-2 c_{1}-\chi$. The complete spectrum is presented in table 2 . 


\begin{tabular}{|c|c|c|c|c|}
\hline $\mathrm{SO}(10) \supset \mathrm{SU}(5)$ & $\mathrm{U}(1)_{i}$ & Homology $(\mathrm{SU}(5))$ & $\mathrm{U}(1)_{Y}$-flux & $\mathrm{U}(1)_{X}$ \\
\hline $16_{t_{1}} \supset 10^{(1)}$ & $t_{1,2}$ & $\eta-2 c_{1}-\chi$ & $-N$ & $M_{16}^{1}$ \\
\hline $16_{t_{3}} \supset 10^{(2)}$ & $t_{3}$ & $-c_{1}+\chi_{5}$ & $N_{5}$ & $M_{16}^{3}$ \\
\hline $16_{t_{4}} \supset 10^{(3)}$ & $t_{4}$ & $-c_{1}+\chi_{7}$ & $N_{7}$ & $M_{16}^{4}$ \\
\hline $45_{t_{5}} \supset 10^{(4)}$ & $t_{5}$ & $-c_{1}+\chi_{9}$ & $N_{9}$ & $C$ \\
\hline $10_{t_{3}+t_{4}} \supset 5^{(0)}$ & $-t_{1}-t_{2}$ & $-c_{1}+\chi$ & $N$ & $M_{10}^{b}+P$ \\
\hline $10_{t_{1}+t_{4}} \supset 5^{(1)}$ & $-t_{1,2}-t_{3}$ & $\eta-2 c_{1}-\chi$ & $-N$ & $-M_{10}^{a}+M-P$ \\
\hline $10_{t_{1}+t_{4}} \supset 5^{(2)}$ & $-t_{1,2}-t_{4}$ & $\eta-2 c_{1}-\chi$ & $-N$ & $M_{10}^{a}+M-P$ \\
\hline $16_{t_{1}} \supset 5^{(3)}$ & $-t_{1,2}-t_{5}$ & $\eta-2 c_{1}-\chi$ & $-N$ & $-M_{16}^{1}+M-P$ \\
\hline $10_{t_{3}+t_{4}} \supset 5^{(4)}$ & $-t_{3}-t_{4}$ & $-c_{1}+\chi-\chi_{9}$ & $N-N_{9}$ & $-M_{10}^{b}+P$ \\
\hline $16_{t_{3}} \supset 5^{(5)}$ & $-t_{3}-t_{5}$ & $-c_{1}+\chi-\chi_{7}$ & $N-N_{7}$ & $-M_{16}^{3}+P_{5}$ \\
\hline $16_{t_{4}} \supset 5^{(6)}$ & $-t_{4}-t_{5}$ & $-c_{1}+\chi-\chi_{5}$ & $N-N_{5}$ & $-M_{16}^{4}+P_{7}$ \\
\hline
\end{tabular}

Table 2. Field representation content under $\mathrm{SU}(5),\left(\mathcal{Z}_{2}\right.$ case) their homology class and flux restrictions under $\mathrm{U}(1)_{Y}$. Note that $\chi=\chi_{5}+\chi_{7}+\chi_{9}$ so the $\mathrm{U}(1)_{Y}$ fluxes satisfy $N=N_{7}+N_{8}+N_{9}$. The last column shows the multiplicities imposed under $\mathrm{U}(1)_{X}$. The $\mathrm{U}(1)_{X}$ fluxes determine the unspecified $M_{i}$ 's of $\mathrm{SU}(5)$ model, while if we assume no chirality in the $\mathrm{SO}(10)$ bulk, then $C=0$ and $P_{5}+P_{7}=P$.

\begin{tabular}{|c|c|c|c|c|}
\hline Matter & Section & Homology & $t_{i}$ charges & $\mathrm{U}(1)_{X}$ \\
\hline $\mathbf{1 6}_{a}$ & $a_{1}$ & $\eta-2 c_{1}-\chi$ & $t_{1,2}$ & $M-P$ \\
$\mathbf{1 6}_{b}$ & $a_{4}$ & $\chi$ & $t_{3,4}$ & $P-C$ \\
$\mathbf{1 0}_{a}$ & $a_{1}-\lambda a_{4}$ & $\eta-2 c_{1}-\chi$ & $t_{1,2}+t_{3,4}$ & $M-P$ \\
$\mathbf{1 0}_{b}$ & $a_{5}$ & $\chi-c_{1}$ & $t_{3}+t_{4} / t_{1}+t_{2}$ & $P$ \\
\hline
\end{tabular}

Table 3. The properties of the $\mathrm{SO}(10)$ representations in the $\mathcal{Z}_{2} \times \mathcal{Z}_{2}$ case.

\section{$2.2 \mathcal{Z}_{2} \times \mathcal{Z}_{2}$ monodromy}

A second class of models arises from the case of $\mathcal{C}_{2+2}$ factorization. In this case we write the spectral cover equation as follows

$$
\mathcal{C}_{4}=\left(a_{3} s^{2}+a_{2} s+a_{1}\right)\left(a_{6} s^{2}+a_{5} s+a_{4}\right)
$$

This splitting implies the $t_{1} \leftrightarrow t_{2}$ and $t_{3} \leftrightarrow t_{4}$ identifications. Comparing with the coefficients $b_{k}$ as in the $\mathcal{Z}_{2}$ case we determine the homologies of $a_{i}$ given in table 26 of the appendix and the properties of the matter curves given in table 3 .

Decomposing the $\mathrm{SO}(10)$ representations to $\mathrm{SU}(5)$ multiplets we get the following multiplicities. For the 16's

$$
\begin{aligned}
\mathbf{1 6}_{t_{1,2}} & = \begin{cases}10_{t_{1,2}} & M_{16}^{1} \\
\overline{5}_{t_{1,2}+t_{5}} & M_{16}^{1}-(M-P) \\
1_{t_{1}-t_{5}} & M_{16}^{1}+(M-P)\end{cases} \\
\mathbf{1 6}_{t_{3,4}}= & \begin{cases}10_{t_{3,4}} & M_{16}^{3} \\
\overline{5}_{t_{3,4}+t_{5}} & M_{16}^{3}-(P-C) \\
1_{t_{3}-t_{5}} & M_{16}^{3}+(P-C)\end{cases}
\end{aligned}
$$


The 10's decompose to $5_{2}+\overline{5}_{-2}$. The following SU(5) originate from their decomposition

$$
\begin{aligned}
\mathbf{1 0}_{t_{1}+t_{2}, t_{3}+t_{4}} & \rightarrow \begin{cases}5_{t_{1}+t_{2}} & M_{10}^{b}+P \\
\overline{5}_{t_{3}+t_{4}} & M_{10}^{b}-P\end{cases} \\
\mathbf{1 0}_{t_{1}+t_{3}, t_{2}+t_{4}} & \rightarrow \begin{cases}5_{t_{1}+t_{3}} & M_{10}^{a}+(M-P) \\
\overline{5}_{t_{2}+t_{4}} & M_{10}^{a}-(M-P)\end{cases}
\end{aligned}
$$

Because of the $t_{1} \leftrightarrow t_{2}$ and $t_{3} \leftrightarrow t_{4}$ monodromies the indices of the fiveplets resulting from $\mathbf{1 0}_{b}$ can be identified $t_{1}+t_{3}=t_{2}+t_{4}$. Therefore we can assign the same indices while

$$
\# 5_{t_{1}+t_{3}}-\# \overline{5}_{t_{1}+t_{3}}=2(M-P)
$$

\subsubsection{SU(5) spectrum}

In the $\mathcal{Z}_{2} \times \mathcal{Z}_{2}$ case, the spectral cover equation for $\mathrm{SU}(5)$ obtains the form

$$
\mathcal{C}_{5}(s)=\left(a_{3} s^{2}+a_{2} s+a_{1}\right)\left(a_{6} s^{2}+a_{5} s+a_{4}\right)\left(a_{7}+a_{8} s\right)
$$

Proceeding as in the $\mathrm{SO}(10)$ case, we identify the relations $b_{k}\left(a_{i}\right), k=1, \ldots 5$ by comparing coefficients of the same power in $s$. The homology classes of $a_{i}$ are given in table 28.

The $10 \in \mathrm{SU}(5)$ are obtained from the solutions of the equation $b_{5}=a_{1} a_{4} a_{7}=0$ therefore they are associated to $a_{1}=0, a_{4}=0$ and $a_{7}=0$. The fiveplets are found by solving the corresponding equation (2.15) and in terms of the $a_{i}$ 's we can write

$$
\begin{aligned}
P_{5}= & \left(a_{6} a_{7}+a_{5} a_{8}\right) \times\left(a_{1}^{2}-a_{1}\left(a_{5} a_{7}+2 a_{4} a_{8}\right) \lambda+a_{4}\left(a_{6} a_{7}^{2}+a_{8}\left(a_{5} a_{7}+a_{4} a_{8}\right)\right) \lambda^{2}\right) \\
& \times\left(a_{1}-a_{5} a_{7} \lambda\right) \times\left(a_{6} a_{7}^{2}+a_{8}\left(a_{5} a_{7}+a_{4} a_{8}\right)\right) \times a_{5}
\end{aligned}
$$

This equation has five factors corresponding to an equal number of fiveplets dubbed as

$$
5^{(0)}, 5^{(1)}, 5^{(2)}, 5^{(3)}, 5^{(4)}
$$

in the order of appearance in the above product. It is straightforward to determine their homology classes using the results of table 28 in the appendix. To compute the flux restrictions we define

$$
\mathcal{F}_{Y} \cdot \psi=-N_{1}-N_{2}, \quad \mathcal{F}_{Y} \cdot \chi=N_{2}, \quad \mathcal{F}_{Y} \cdot \eta=\mathcal{F}_{Y} \cdot c_{1}=0
$$

The results are summarized in table 4 .

\section{$2.3 \quad \mathcal{Z}_{3}$ monodromy}

We write the spectral cover equation as follows

$$
\mathcal{C}_{4}=\left(a_{1}+a_{2} s+a_{3} s^{2}+a_{4} s^{3}\right)\left(a_{5}+s a_{6}\right)
$$

Comparing with the coefficients $b_{k}$, we determine as previously the homologies of $a_{i}$ 's. We may choose two different ways to solve the $b_{1}=0$ constraint, either $a_{5}=\lambda a_{6}, a_{3}=-\lambda a_{4}$ or $a_{3}=-\lambda a_{5}, a_{4}=\lambda a_{6}$. The homology classes of $a_{i}$ are shown in table 29 of the appendix.

Proceeding as in the previous cases we determine the homologies of 16, 10 matter curves. In particular from the equation $b_{4}=a_{1} a_{5}$ we infer that the homologies of $\mathbf{1 6}$ 's are $\eta-3 c_{1}-\chi$ and $\chi-c_{1}$ respectively, while for those of the 10's we choose them in consistency of the defining equation $b_{3}^{2}=0$. The results are collected in table 5. For the second Ansatz the properties of the 10-representations change according to table 6 . 


\begin{tabular}{|c|c|c|c|c|}
\hline $\mathrm{SO}(10) \supset \mathrm{SU}(5)$ & $\mathrm{U}(1)_{i}$ & Homology $(\mathrm{SU}(5))$ & $\mathrm{U}(1)_{Y}$-flux & $\mathrm{U}(1)_{X}$ \\
\hline $16_{t_{1}} \supset 10^{(1)}$ & $t_{1,2}$ & $\eta-2 c_{1}-\chi-\psi$ & $N_{1}$ & $M_{16}^{1}$ \\
\hline $16_{t_{3}} \supset 10^{(2)}$ & $t_{3}$ & $-2 c_{1}+\chi$ & $N_{2}$ & $M_{16}^{3}$ \\
\hline $10_{t_{3}+t_{4}} \supset 5^{(0)}$ & $-t_{1}-t_{2}$ & $-c_{1}+\chi+\psi$ & $-N_{1}$ & $M_{10}^{b}+P$ \\
\hline $10_{t_{1}+t_{4}} \supset 5^{(1)}$ & $-t_{1,2}-t_{3}$ & $2 \eta-4 c_{1}-2 \chi-2 \psi$ & $2 N_{1}$ & $2(M-P)$ \\
\hline $16_{t_{1}} \supset 5^{(2)}$ & $-t_{1,2}-t_{5}$ & $\eta-2 c_{1}-\chi-\psi$ & $N_{1}$ & $-M_{16}^{1}+M-P$ \\
\hline $16_{t_{3}} \supset 5^{(3)}$ & $-t_{3,4}-t_{5}$ & $-2 c_{1}+\chi+\psi$ & $-2 N_{1}-N_{2}$ & $-M_{16}^{3}+P-C$ \\
\hline $10_{t_{3}+t_{4}} \supset 5^{(4)}$ & $-t_{3}-t_{4}$ & $-c_{1}+\chi$ & $N_{2}$ & $-M_{10}^{b}+P$ \\
\hline $45 \supset 10^{(3)}$ & $t_{5}$ & $-c_{1}+\psi$ & $-N_{1}-N_{2}$ & $M_{10}^{c}$ \\
\hline
\end{tabular}

Table 4. Field representation content under SU(5), their homology class and flux restrictions under $\mathrm{U}(1)_{Y}$ for the $\mathcal{Z}_{2} \times \mathcal{Z}_{2}$ case.

\begin{tabular}{|c|c|c|c|c|}
\hline Matter & Section & Homology & $t_{i}$ charges & $\mathrm{U}(1)_{X}$ \\
\hline $\mathbf{1 6}$ & $a_{1}$ & $\eta-3 c_{1}-\chi$ & $t_{i}$ & $M-P+C$ \\
$\mathbf{1 6}$ & $a_{5}$ & $\chi-c_{1}$ & $t_{4}$ & $P$ \\
$\mathbf{1 0}$ & $a_{1}+\lambda a_{2}$ & $\eta-3 c_{1}-\chi$ & $2 t_{i}$ & $M-P+C$ \\
$\mathbf{1 0}$ & $a_{6}$ & $\chi$ & $t_{i}+t_{4}$ & $P-C$ \\
\hline
\end{tabular}

Table 5. $\mathcal{Z}_{3}$ case $A$. Properties of $\mathrm{SO}(10)$ representations (with $i$ any of $1,2,3$ ).

\begin{tabular}{|c|c|c|c|c|}
\hline Matter & Section & Homology & $t_{i}$ charges & $\mathrm{U}(1)_{X}$ \\
\hline $\mathbf{1 6}$ & $a_{1}$ & $\eta-3 c_{1}-\chi$ & $t_{i}$ & $M-P+C$ \\
$\mathbf{1 6}$ & $a_{5}$ & $\chi-c_{1}$ & $t_{4}$ & $P$ \\
$\mathbf{1 0}$ & $a_{1} a_{6}+a_{2} a_{5}$ & $\eta-3 c_{1}$ & $2 t_{i}$ & $M$ \\
$\mathbf{1 0}$ & $a_{1} a_{6}+a_{2} a_{5}$ & $\eta-3 c_{1}$ & $t_{i}+t_{4}$ & $M$ \\
\hline
\end{tabular}

Table 6. $\mathcal{Z}_{3}$ case $B$. Properties of $\mathrm{SO}(10)$ representations (with $i$ any of $1,2,3$ ).

\subsubsection{SU(5) spectrum}

In this case the relevant spectral cover polynomial is

$$
\sum_{k=0}^{5} b_{k} s^{5-k}=\left(a_{4} s^{3}+a_{3} s^{2}+a_{2} s+a_{1}\right)\left(a_{5}+s a_{6}\right)\left(a_{7}+s a_{8}\right)
$$

We can easily extract the equations determining the coefficients $b_{k}\left(a_{i}\right)$, while the corresponding one for the homologies reads

$\left[b_{k}\right]=\eta-k c_{1}=\left[a_{l}\right]+\left[a_{m}\right]+\left[a_{n}\right], \quad k=0,1, \ldots, 5, \quad k+l+m+n=18, \quad l, m, n \leq 8$

The solution $b_{1}=0$ and other details are shown in the appendix. The tenplets are determined by $b_{5}=a_{1} a_{5} a_{7}=0$, while the equation (2.15) factorizes as follows

$$
\begin{aligned}
P_{5}= & \left(a_{1} a_{6} a_{8}+a_{2}\left(a_{6} a_{7}+a_{5} a_{8}\right)\right) \times\left(a_{1} a_{6}+a_{5}\left(a_{2}-c a_{5} a_{7}\right)\right) \\
& \times\left(a_{7}\left(a_{2}-c a_{5} a_{7}\right)+a_{1} a_{8}\right) \times\left(a_{6} a_{7}+a_{5} a_{8}\right)
\end{aligned}
$$




\begin{tabular}{|c|c|c|c|c|}
\hline $\mathrm{SO}(10) \supset \mathrm{SU}(5)$ & $\mathrm{U}(1)_{i}$ & Homology $(\mathrm{SU}(5))$ & $\mathrm{U}(1)_{Y}$-flux & $\mathrm{U}(1)_{X}$ \\
\hline $16_{t_{i}} \supset 10^{(1)}$ & $t_{i}$ & $\eta-3 c_{1}-\chi-\psi$ & $-N_{x}-N_{y}$ & $M_{16}^{1}$ \\
\hline $16_{t_{4}} \supset 10^{(2)}$ & $t_{4}$ & $-c_{1}+\chi$ & $N_{x}$ & $M_{16}^{2}$ \\
\hline $10_{t_{i}+t_{4}} \supset 5^{(0)}$ & $-2 t_{i}$ & $\eta-3 c_{1}$ & 0 & $M_{10}+n M$ \\
\hline $10_{t_{i}+t_{j}} \supset 5^{(1)}$ & $-t_{i}-t_{4}$ & $\eta-3 c_{1}-\psi$ & $-N_{y}$ & $-M_{10}+n M$ \\
\hline $16_{t_{i}} \supset 5^{(2)}$ & $-t_{i}-t_{5}$ & $\eta-3 c_{1}-\chi$ & $-N_{x}$ & $-M_{16}^{1}+(M-P+C)$ \\
\hline $16_{t_{4}} \supset 5^{(3)}$ & $-t_{4}-t_{5}$ & $-c_{1}+\chi+\psi$ & $N_{x}+N_{y}$ & $-M_{16}^{2}+P$ \\
\hline $45 \supset 10^{(3)}$ & $t_{5}$ & $-c_{1}+\psi$ & $N_{y}$ & $M_{10}^{\prime}$ \\
\hline
\end{tabular}

Table 7. Field representation content under SU(5), their homology class and flux restrictions with respect to $\mathrm{U}(1)_{Y}$ for the $\mathcal{Z}_{3}$ case. The coefficient $n$ takes the values 1 for case $A$ and 2 for case $B$.

The four factors determine the homologies of the fiveplets dubbed $5^{(0)}, 5^{(1)}, 5^{(2)}, 5^{(3)}$ correspondingly. These, together with the tenplets are given in table 7 .

Decomposition of 10s of $\mathrm{SO}(10)$ in more subtle. Observe first that $10_{t_{k}+t_{4}}$ and eventually their complex conjugates $\overline{10}_{-t_{k}-t_{4}}$ sit on a matter curve (dubbed here $\Sigma_{10_{1}}$ ). Also $10_{t_{i}+t_{j}}$ and their complex conjugates are on $\Sigma_{10_{2}}$. Notice however that $t_{i}+t_{j}+t_{k}+t_{4}=0$, so that $\overline{10}_{-t_{k}-t_{4}}=10_{t_{i}+t_{j}}$, i.e., the two curves accommodate fields with the same $\mathrm{U}(1)_{t_{a}}$ charges.

Adopting $t_{1,2,3}=t_{i}$ we have

$$
\mathbf{1 0}_{t_{1}+t_{3}} \rightarrow \mathbf{1 0}_{2 t_{i},-t_{i}-t_{4}} \rightarrow \begin{cases}5_{-t_{i}-t_{4}} & M_{10}^{a}+Q \\ \overline{5}_{2 t_{i}} & M_{10}^{a}-Q\end{cases}
$$

where $Q=(M-P+C)$ for the first model and $Q=M$ for the second one. Also

$$
\mathbf{1 0}_{t_{1}+t_{4}} \rightarrow \mathbf{1 0}_{t_{1}+t_{4},-2 t_{i}} \rightarrow \begin{cases}5_{-2 t_{i}} & M_{10}^{b}+U \\ \overline{5}_{t_{i}+t_{4}} & M_{10}^{b}-U\end{cases}
$$

with $U=P-C$ and $U=M$ respectively.

At the $\mathrm{SU}(5)$ level we have only two distinct species of fiveplets emerging from $\mathbf{1 0}$ of $\mathrm{SO}(10)$ and the differences are given $\left(M_{10}=M_{10}^{b}-M_{10}^{a}\right)$

$$
\begin{aligned}
\# 5_{-2 t_{i}}-\# \overline{5}_{2 t_{i}} & =M_{10}+V \\
\# 5_{-t_{i}-t_{4}}-\# \overline{5}_{t_{i}+t_{4}} & =-M_{10}+V
\end{aligned}
$$

where $V=Q+U=n M$ with $n=1,2$ for the first and second case respectively.

\section{Low energy effective models}

Once we have obtained the properties of matter curves and the necessary ingredients we can start our investigation for a viable effective low energy theory emerging from the above picture. To discuss low energy effective models of $\mathrm{SO}(10)$ origin, we further need to specify the symmetry breaking mechanism down to the Standard Model. We will assume 
here that $\mathrm{SO}(10)$ breaks to $\mathrm{SU}(5)$ by flux along $\mathrm{U}(1)_{X}$. At the next stage of symmetry breaking a $\mathrm{U}(1)_{Y}$ flux must be turned on. In the subsequent we need to take into account the hypercharge splitting. When $\mathrm{SO}(10)$ breaks by fluxes, we have already seen how the $\mathrm{U}(1)_{X}$ flux restricts on the relevant matter curves providing information for the chiral $\mathrm{SU}(5)$ spectrum. In a similar way, $\mathrm{U}(1)_{Y}$ flux splits the $\mathrm{SU}(5)$ multiplets.

Assuming that the integer $N_{Y}$ represents the effect of the $\mathrm{U}(1)_{Y}$ hypercharge flux on a specific matter curve and that the $\mathrm{U}(1)_{X}$ flux is given by the integers $M_{10,5}$ the resulting SM spectrum appears with the following multiplicities:

$$
\mathbf{1 0} \in \mathrm{SU}(5) \Rightarrow\left\{\begin{array}{l}
n_{(3,2)_{\frac{1}{6}}}-n_{(\overline{3}, 2)_{-\frac{1}{6}}}=M_{10} \\
n_{(\overline{3}, 1)_{-\frac{2}{3}}}-n_{(3,1)_{\frac{2}{3}}}=M_{10}-N_{Y} \\
n_{(1,1)_{1}}-n_{(1,1)_{-1}}=M_{10}+N_{Y}
\end{array}\right.
$$

and

$$
\mathbf{5} \in \mathrm{SU}(5) \Rightarrow\left\{\begin{array}{l}
n_{(3,1)_{-\frac{1}{3}}}-n_{(\overline{3}, 1)_{\frac{1}{3}}}=M_{5} \\
n_{(1,2)_{\frac{1}{2}}}-n_{(1,2)_{-\frac{1}{2}}}=M_{5}+N_{Y}
\end{array}\right.
$$

In our subsequent analysis we will use these formulae repeatedly for each matter curve to determine the SM matter spectrum.

In order to break the $\mathrm{SO}(10)$ and $\mathrm{SU}(5)$ GUT symmetries in the present constructions we have appealed to the generic mechanism of abelian fluxes. We have observed that, in addition to inducing the symmetry breaking, such fluxes also determine the zero-mode spectrum of the low energy theory. Zero mode fields eventually may arise in the intersections of the GUT brane with other seven branes as well as in the bulk. Then, the anticipated chiral and Higgs Standard Model spectrum could emerge either from the bulk modes or from the representations residing on the matter curves or from both sources.

In the case of the $\mathrm{SU}(5)$ breaking down to $\mathrm{SM}$, the decomposition of the adjoint representation involves the bulk states $(3,2)_{-5},(\overline{3}, 2)_{5}$ which carry exotic charges. These can be eliminated by the well known conditions [3, 4]

$$
c_{1}\left(\mathcal{L}_{Y}^{5}\right) \cdot c_{1}\left(\mathcal{L}_{Y}^{5}\right)=-2 \quad \text { and } \quad c_{1}\left(\mathcal{L}_{Y}^{5}\right) \cdot c_{1}(\mathcal{S})=0
$$

For the $\mathrm{SO}(10)$ case, extraneous matter arising from the decomposition of the adjoint, i.e. 45, falls into $10_{4}$ and $\overline{10}_{-4}$ representations of $\mathrm{SU}(5)$. If the latter come in vector-like pairs, appropriate large singlet fields could develop vevs so that that such pairs will develop a large mass and get lifted from the low energy spectrum. Indeed, for a line-bundle $\mathcal{L}_{X}$ on a del Pezzo surface $S$ the condition

$$
c_{1}\left(\mathcal{L}_{X}\right) \cdot c_{1}(\mathcal{S})=0
$$

ensures the appearance only of vector-like pairs of bulk states associated to the line bundle $\mathcal{L}_{X}$.

In the most general case, one should consider the representations obtained from the decompositions of the $\mathbf{4 5}$, the $\mathbf{1 6}+\overline{\mathbf{1 6}}$ and $\mathbf{1 0}+\overline{\mathbf{1 0}}$ representations of $\mathrm{SO}(10)$. For a direct $\mathrm{SO}(10)$ breaking down to the SM gauge symmetry one can prove that it is impossible [5] 
to avoid all extraneous matter, however it is still possible to satisfy the restrictions (3.3) to obtain the latter in vector like pairs.

One may finally consider the case of the extraneous states by turning on the gluing morphism $[40,41]$. Recall that under $\mathrm{SO}(10)$ breaking down to $\mathrm{SU}(5) \times \mathrm{U}(1)_{X}$ there appears a singlet charged under $\mathrm{U}(1)_{X}$ according to $\mathbf{1 6} \rightarrow 10_{-1}+\overline{5}_{3}+1_{-5}$ and analogously for $\overline{\mathbf{1 6}}$. Then the gluing morphism is realised when the singlet field $\theta_{5 i} \equiv \overline{1}_{5}$ develops a non-zero vev so that bulk modes $\overline{10}_{-4}$ can couple to matter curve states through terms of the form $\overline{10}_{-4} 10_{-1} \overline{1}_{5}$. Further, it is possible to accommodate some chiral matter in the bulk and generate interesting hierarchies [41].

In what follows, we will only consider cases where chiral matter is localised only along the matter curves. Then, the expected fermion mass hierarchy will be attributed either to the non-commutative geometry [25] or to the non-renormalisable contributions in the superpotential through the Froggatt-Nielsen mechanism [43].

\section{$3.1 \mathcal{Z}_{2}$ models}

For the case of $\mathcal{Z}_{2}$ monodromy we have already given the properties of the $\mathrm{SO}(10)$ spectrum in table 1. All properties of the SU(5) representations are summarized in table 2.

In previous studies in the context of the $\mathrm{SU}(5)$ level, the $M_{5_{i}}, M_{10_{j}}$ are not fully determined. It is a fact however that there is a non-trivial connection between the $\mathrm{U}(1)_{i}$ symmetries and the flux. One condition on $M_{i}$ 's can be imposed by demanding the U(1)'s to be traceless. This implies $[21,44]$

$$
\sum M_{10_{j}}+\sum_{i} M_{5_{i}}=0
$$

Interestingly, this condition can be recovered by appealing to the properties of a specific $\mathrm{U}(1)$. In particular if we assume the embedding of $\mathrm{SU}(5)$ in $\mathrm{SO}(10) \supset \mathrm{SU}(5) \times \mathrm{U}(1)_{X}$, the associated $\mathrm{U}(1)_{X}$ flux implies restrictions on $M_{i}$ 's. The relation (3.4) can be derived under the following two assumptions:

$$
\mathcal{F}_{1} \cdot \eta=0 \quad \text { and } \quad \mathcal{F}_{1} \cdot c_{1}=0
$$

which imply that both flux parameters $M, C$ are zero $M=C=0$. Note that these conditions are equivalent to those imposed to the $\mathrm{U}(1)_{Y}$ flux. As in that case, they ensure that the $\mathrm{U}(1)_{X}$ boson does not get a GS term. Thus, adding all $M_{i}^{\prime} s$ from table 2 while respecting the above conditions we get

$$
\sum_{i} M_{i}=0
$$

since $P=P_{5}+P_{7}$ and $M=0$. Thus, this is the equivalent to the relation (3.4). Notice however that $\mathrm{U}(1)_{X}$ flux imposes additional relations among $M_{i}$ 's as can be seen from the last column of table 1 .

Our main goal in this section is to present specific models with realistic properties and spectra, combining the flux constraints emerging from the successive flux breaking. In 
order to assess the implications of these combined data, we will compare our findings with the $\mathrm{SU}(5)$ models already discussed in the literature.

A class of models may arise as follows: we have seen that assuming a $\mathcal{Z}_{2}$ monodromy, the matter curves accommodating 16's reduce to three and there are four curves left over to accommodate the 10's of $\mathrm{SO}(10)$. Further breaking of the gauge group down to $\mathrm{SU}(5)$ with fluxes, will result to three matter curves $\Sigma_{10_{t_{1}}}, \Sigma_{10_{t_{3}}}, \Sigma_{10_{t_{4}}}$ accommodating the $10 \in \mathrm{SU}(5)$ and seven curves $\Sigma_{5_{i}+t_{j}}$ for the relevant 5's. A fourth curve could also accommodate a $10 \in \mathrm{SU}(5)$ from the bulk adjoint $\mathbf{4 5}$, however we assume that no chirality is generated in the $\mathrm{SO}(10)$ bulk. These are shown in table 2.

We consider the case where at least the third family resides on $\Sigma_{10_{t_{1}}}$ and demand that the mass is obtained at tree level. Then the up-Higgs is found in $\Sigma_{5_{t_{1}+t_{2}}}$

$$
\lambda_{t} 10_{t_{1}} \cdot 10_{t_{2}} \cdot 5_{-t_{1}-t_{2}} \stackrel{\mathcal{Z}_{2}}{\longrightarrow} \lambda_{t} 10_{t_{1}} \cdot 10_{t_{1}} \cdot 5_{-2 t_{1}}
$$

Since up and bottom quarks constitute a left handed doublet they naturally reside on the same matter curve. Demanding that the bottom quark has also a tree-level coupling, the only available is

$$
\lambda_{b} 10_{t_{1}} \cdot \overline{5}_{t_{2}+t_{5}} \cdot \overline{5}_{t_{3}+t_{4}} \stackrel{\mathcal{Z}_{2}}{\longrightarrow} \lambda_{b} 10_{t_{1}} \cdot \overline{5}_{t_{1}+t_{5}} \cdot \overline{5}_{t_{3}+t_{4}}
$$

where one of the fiveplets $\overline{5}_{t_{1}+t_{5}}, \overline{5}_{t_{3}+t_{4}}$ should accommodate the right handed chiral matter and the other the down-type Higgs field.

Now, having in mind the properties of the $\Sigma_{10_{t_{i}}}$ curves with respect the $\mathrm{U}(1)_{Y}$-flux (see relevant column of table 2) we find that a convenient choice of multiplicities is

$$
M_{16}^{1}=4, M_{16}^{3}=0, M_{16}^{4}=-1
$$

These ensure that there exist three $Q=(u, d)^{T}$ doublets to accommodate the corresponding fermionic degrees of freedom and an additional vector like pair $Q+\bar{Q}$. Furthermore the choice

$$
N=N_{9}=0, \quad N_{5}=1, \quad N_{7}=-1
$$

will result to the following matter content:

$$
\begin{aligned}
& \mathbf{1 0}_{t_{1}}=\left\{\begin{array}{l}
n_{(3,2)_{1 / 6}}-n_{(\overline{3}, 2)_{-1 / 6}}=4 \rightarrow 4 \times Q \\
n_{(\overline{3}, 1)_{-2 / 3}}-n_{(3,1)_{2 / 3}}=4 \rightarrow 4 \times u^{c} \\
n_{(1,1)_{1}}-n_{(1,1)_{-1}}=4 \rightarrow 4 \times e^{c}
\end{array}\right. \\
& \mathbf{1 0}_{t_{3}}= \begin{cases}n_{(3,2)_{1 / 6}}-n_{(\overline{3}, 2)_{-1 / 6}}=0 & \rightarrow 0 \times Q \\
n_{(\overline{3}, 1)_{-2 / 3}}-n_{(3,1)_{2 / 3}}=-1 & \rightarrow 1 \times \bar{u}^{c} \\
n_{(1,1)_{1}}-n_{(1,1)_{-1}}=1 & \rightarrow 1 \times e^{c}\end{cases} \\
& \mathbf{1 0}_{t_{4}}= \begin{cases}n_{(3,2)_{1 / 6}}-n_{(\overline{3}, 2)_{-1 / 6}}=-1 \rightarrow 1 \times \bar{Q} \\
n_{(\overline{3}, 1)_{-2 / 3}}-n_{(3,1)_{2 / 3}}=0 \rightarrow 0 \times \bar{u}^{c} \\
n_{(1,1)_{1}}-n_{(1,1)_{-1}}=-2 & \rightarrow 2 \times \bar{e}^{c}\end{cases}
\end{aligned}
$$

The first matter curve $10^{(1)}$ accommodates four complete $10_{t_{1}}$ 's of SU(5). On the second curve $10^{(2)}$ we obtain $\bar{Q}$ from an incomplete $\overline{10}_{-t_{3}}$ and only an $e^{c}$ state from the corresponding $10_{t_{3}}$. Finally, $10^{(3)}$ provides $\bar{Q}+2 \bar{e}^{c}$ from $\overline{10}_{t_{4}}$ 's. Therefore, we end up with 


\begin{tabular}{|c|c|c|c|}
\hline SU(5) curves & $\mathrm{U}(1)_{i}$ & $\mathrm{U}(1)_{Y^{-}}$flux & $\mathrm{U}(1)_{X}$ \\
\hline$\Sigma_{10^{(1)}}$ & $t_{1,2}$ & 0 & 4 \\
\hline$\Sigma_{10^{(2)}}$ & $t_{3}$ & 1 & 0 \\
\hline$\Sigma_{10^{(3)}}$ & $t_{4}$ & -1 & -1 \\
\hline$\Sigma_{10^{(4)}}$ & $t_{5}$ & 0 & 0 \\
\hline$\Sigma_{5^{(0)}}$ & $-t_{1}-t_{2}$ & 0 & $M_{10}^{b}+P$ \\
\hline$\Sigma_{5^{(1)}}$ & $-t_{1,2}-t_{3}$ & 0 & $-M_{10}^{a}+M-P$ \\
\hline$\Sigma_{5^{(2)}}$ & $-t_{1,2}-t_{4}$ & 0 & $M_{10}^{a}+M-P$ \\
\hline$\Sigma_{5^{(3)}}$ & $-t_{1,2}-t_{5}$ & 0 & $-4+M-P$ \\
\hline$\Sigma_{5^{(4)}}$ & $-t_{3}-t_{4}$ & 0 & $-M_{10}^{b}+P$ \\
\hline$\Sigma_{5^{(5)}}$ & $-t_{3}-t_{5}$ & 1 & $P_{5}$ \\
\hline$\Sigma_{5^{(6)}}$ & $-t_{4}-t_{5}$ & -1 & $1+P_{7}$ \\
\hline
\end{tabular}

Table 8. Field representation with three families and extra vector like pairs $Q+\bar{Q}, u^{c}+\bar{u}^{c}$ and two $e^{c}+\bar{e}^{c}$ in $\mathcal{Z}_{2}$ case.

$3 \times\left(Q+u^{c}+e^{c}\right)$, one pair $Q+\bar{Q}$ and $u^{c}+\bar{u}^{c}$ and two pairs $e^{c}+\bar{e}^{c}$. The spectrum is shown in table 8 .

The choice of the flux parameters should secure the existence of three $5_{f}$ 's and the Higgs doublets $h_{u}+h_{d}$. A constraint emerges then from the fact that the number of triplets minus antitriplets should be -3 . To this end we impose

$\left(M_{10}^{b}+P\right)+\left(M-P-M_{10}^{a}\right)+\left(M_{10}^{a}+M-P\right)+(M-P-4)+\left(P-M_{10}^{b}\right)+\left(P_{5}+1+P_{7}\right)=-3$

which is automatically satisfied for $M=C=0$. Notably, this is equivalent to the assumptions in (3.5). Next we use the freedom to adjust the remaining flux parameters $M_{10}^{i}, P_{j}$ to obtain the appropriate 5 and $\overline{5}$ representations. There are several options and in the subsequent analysis we discuss a few of them.

\subsubsection{Model $A$}

We start our investigation by imposing a condition to ensure the up-type Higgs $h_{u}$. Since we need at least one $h_{u}$, the simplest possibility is

$$
M_{10}^{b}+P=1 \rightarrow M_{10}^{b}=1-P
$$

while we take $M=C=N=M_{10}^{a}=0$. Because $N=0$, the Higgs fiveplet living on $5^{(0)}$ does not split. The same happens also for fiveplets eventually residing on $5^{(1,2,3,4)}$ curves. Splitting occurs only on matter curves $5^{(5,6)}$. In particular, we have

$$
5^{(5)}=\left\{\begin{array}{l}
n_{(3,1)_{-1 / 3}}-n_{(\overline{3}, 1)_{1 / 3}}=P_{5} \\
n_{(1,2)_{1 / 2}}-n_{(1,2)_{-1 / 2}}=P_{5}+1
\end{array}, \quad 5^{(6)}=\left\{\begin{array}{l}
n_{(3,1)_{-1 / 3}}-n_{(\overline{3}, 1)_{1 / 3}}=1+P-P_{5} \\
n_{(1,2)_{1 / 2}}-n_{(1,2)_{-1 / 2}}=P-P_{5}
\end{array}\right.\right.
$$

Another constraint arises from the fact that the down-Higgs should reside in $\overline{5}^{(4)}$

$$
2 P-1<0 \rightarrow P \leq 0
$$




\begin{tabular}{|c|c|c|}
\hline Curves & $\mathrm{U}(1)_{i}$ & SM matter content \\
\hline$\Sigma_{10^{(1)}}$ & $t_{1,2}$ & $4 \times\left(Q+u^{c}+e^{c}\right)$ \\
\hline$\Sigma_{10^{(2)}}$ & $t_{3}$ & $1 \times\left(\bar{u}^{c}+e^{c}\right)$ \\
\hline$\Sigma_{\overline{10}(3)}$ & $t_{4}$ & $1 \times \bar{Q}+2 \times \bar{e}^{c}$ \\
\hline$\Sigma_{10^{(4)}}$ & $t_{5}$ & 0 \\
\hline$\Sigma_{5^{(0)}}$ & $-t_{1}-t_{2}$ & $1 \times\left(h_{u}+D\right)$ \\
\hline$\Sigma_{5^{(1)}}$ & $-t_{1,2}-t_{3}$ & 0 \\
\hline$\Sigma_{5^{(2)}}$ & $-t_{1,2}-t_{4}$ & 0 \\
\hline$\Sigma_{5^{(3)}}$ & $-t_{1,2}-t_{5}$ & $4 \times\left(d^{c}+\ell\right)$ \\
\hline$\Sigma_{5^{(4)}}$ & $-t_{3}-t_{4}$ & $1 \times\left(\bar{D}+h_{d}\right)$ \\
\hline$\Sigma_{5^{(5)}}$ & $-t_{3}-t_{5}$ & $P_{5} \times D+\left(1+P_{5}\right) \times h$ \\
\hline$\Sigma_{5^{(6)}}$ & $-t_{4}-t_{5}$ & $\left(1-P_{5}\right) \times D+\left(-P_{5}\right) \times h$ \\
\hline
\end{tabular}

Table 9. Case A of $\mathcal{Z}_{2}$ : field representation of SU(5) matter curves (first column). Last column shows the multiplicities of the SM representations.

Assuming the minimal case $P=0$, we get

$$
\overline{5}^{(4)} \rightarrow 1 \times\left(\bar{D}, h_{d}\right)
$$

Table 9 summarizes the spectrum where the only parameter left unspecified up to now is $P_{5}$. Different choices of $P_{5}$ affect the way $\Sigma_{5(5,6)}$ split leading to different effective models.

The following Yukawa couplings emerge involving the 10's:

$$
\begin{aligned}
10^{(1)} 10^{(1)} 5^{(0)} & \rightarrow Q u^{c} h_{u} \\
10^{(1)} 10^{(2)} 5^{(0)} \theta_{13} & \rightarrow u^{c} e^{c} D\left\langle\theta_{13}\right\rangle \\
10^{(1)} \overline{10}^{(2)} \theta_{31} & \rightarrow \bar{u}^{c} u^{c}\left\langle\theta_{31}\right\rangle \\
10^{(1)} \overline{10} \overline{10}^{(3)} \theta_{41} & \rightarrow\left(\bar{Q} Q+\bar{e}^{c} e^{c}\right)\left\langle\theta_{41}\right\rangle
\end{aligned}
$$

Choosing $P_{5}=1$, the last two 5's give

$$
\begin{aligned}
& 5^{(5)}=1 \times D+2 \times h_{u}^{\prime} \\
& \overline{5}^{(6)}=0 \times \bar{D}+1 \times h_{d}^{\prime}
\end{aligned}
$$

In this case, splitting occurs on 5-curves other than the Higgs. The triplets can in principle form the following mass terms

$$
\mathcal{W}_{T} \propto\left(\left\langle\theta_{15}\right\rangle \overline{5}^{(3)}+\left\langle\theta_{13}\right\rangle\left\langle\theta_{14}\right\rangle \overline{5}^{(4)}\right) 5^{(0)}+\left(\left\langle\theta_{31}\right\rangle \overline{5}^{(3)}+\left\langle\theta_{54}\right\rangle \overline{5}^{(4)}\right) 5^{(5)}
$$

and the doublets

$$
\mathcal{W}_{h_{u, d}} \propto\left(\left\langle\theta_{15}\right\rangle \overline{5}^{(3)}+\left\langle\theta_{13}\right\rangle\left\langle\theta_{14}\right\rangle \overline{5}^{(4)}+\left\langle\theta_{15}\right\rangle\left\langle\theta_{14}\right\rangle \overline{5}^{(6)}\right) 5^{(0)}+\left(\left\langle\theta_{31}\right\rangle \overline{5}^{(3)}+\left\langle\theta_{54}\right\rangle \overline{5}^{(4)}+\left\langle\theta_{34}\right\rangle \overline{5}^{(6)}\right) 5^{(5)}
$$

The Higgs doublets mass matrix differs from that of the color triplets, allowing for the possibility to make the latter massive while keeping one Higgs doublet pair light. However, 


\begin{tabular}{|c|c|c|}
\hline Curves & $\mathrm{U}(1)_{i}$ & SM matter content \\
\hline$\Sigma_{10^{(1)}}$ & $t_{1,2}$ & $4 \times\left(Q+u^{c}+e^{c}\right)$ \\
\hline$\Sigma_{10^{(2)}}$ & $t_{3}$ & $1 \times\left(\bar{u}^{c}+e^{c}\right)$ \\
\hline$\Sigma_{\overline{10}(3)}$ & $t_{4}$ & $1 \times \bar{Q}+2 \times \bar{e}^{c}$ \\
\hline$\Sigma_{10^{(4)}}$ & $t_{5}$ & 0 \\
\hline$\Sigma_{5^{(0)}}$ & $-t_{1}-t_{2}$ & $2 \times\left(D, h_{u}\right)$ \\
\hline$\Sigma_{5^{(1)}}$ & $-t_{1,2}-t_{3}$ & $2 \times\left(\bar{D}, h_{d}^{\prime}\right)$ \\
\hline$\Sigma_{5^{(2)}}$ & $-t_{1,2}-t_{4}$ & 0 \\
\hline$\Sigma_{5^{(3)}}$ & $-t_{1,2}-t_{5}$ & $5 \times\left(d^{c}+\ell\right)$ \\
\hline$\Sigma_{5^{(4)}}$ & $-t_{3}-t_{4}$ & 0 \\
\hline$\Sigma_{5^{(5)}}$ & $-t_{3}-t_{5}$ & $2 \times D+3 \times h_{u}^{\prime}$ \\
\hline$\Sigma_{5^{(6)}}$ & $-t_{4}-t_{5}$ & $0 \times D+1 \times h_{d}$ \\
\hline
\end{tabular}

Table 10. Case B of $\mathcal{Z}_{2}$ : field representation of SU(5) matter curves (first column). Last column shows the multiplicities of the SM representations.

a detailed knowledge of Yukawa couplings and possible fine tuning would be needed to make triplets massive while keeping doublets light. Therefore, we proceed to a second set of flux parameters.

\subsubsection{Model $B$}

We can choose the down Higgs doublet to descend from a $\Sigma_{5}$-curve whose matter context splits by flux and derive the bottom coupling from a suitable non-renormalizable (NR)-term.

Choosing in this case $M_{10}^{a}=M_{10}^{b}=P=1, P_{7}=-1, P_{5}=2$, the spectrum is that of table 10. The bottom receives a mass

$$
10^{(1)} \overline{5}^{(3)} \overline{5}^{(6)} \theta_{35}
$$

The triplets can in principle form the following mass terms

$$
\mathcal{W}_{T} \propto\left(\left\langle\theta_{15}\right\rangle \overline{5}^{(3)}+\left\langle\theta_{13}\right\rangle \overline{5}^{(1)}\right) 5^{(0)}+\left(\left\langle\theta_{31}\right\rangle \overline{5}^{(3)}+\left\langle\theta_{51}\right\rangle \overline{5}^{(1)}\right) 5^{(5)}
$$

while the doublets involve additional terms,

$\left.\mathcal{W}_{h_{u, d}} \propto\left(\left\langle\theta_{15}\right\rangle \overline{5}^{(3)}+\left\langle\theta_{13}\right\rangle\right\rangle \overline{5}^{(1)}+\left\langle\theta_{15}\right\rangle\left\langle\theta_{14}\right\rangle \overline{5}^{(6)}\right) 5^{(0)}+\left(\left\langle\theta_{31}\right\rangle \overline{5}^{(3)}+\left\langle\theta_{51}\right\rangle \overline{5}^{(1)}+\left\langle\theta_{34}\right\rangle \overline{5}^{(6)}\right) 5^{(5)}$

A suitable choice of $\theta_{i j}$ vevs can leave one Higgs doublet pair light, while all triplets receive heavy masses.

\subsubsection{Model $C$}

We present now a model with the three families distributed in three different matter curves. We choose $10^{(1)}, 10^{(2)}, 10^{(3)}$ to accommodate the $\left(Q, u^{c}, e^{c}\right)$ SM multiplets, thus $M_{16}^{i}=1$, 


\begin{tabular}{|c|c|c|c|c|c|}
\hline Matter curve & $\mathrm{SU}(5) \times \mathrm{U}(1)_{i}$ & $N_{Y}$ & $M_{X}$ & SM reps & $R$-parity \\
\hline$\Sigma_{10^{(1)}}$ & $10_{t_{1,2}}$ & 0 & 1 & $1 \times\left(Q, t^{c}, \tau^{c}\right)$ & - \\
\hline$\Sigma_{10^{(2)}}$ & $10_{t_{3}}$ & -1 & 1 & $1 \times Q+2 \times u^{c}$ & - \\
\hline$\Sigma_{10^{(3)}}$ & $10_{t_{4}}$ & 1 & 1 & $1 \times Q+2 \times e^{c}$ & - \\
\hline$\Sigma_{5^{(0)}}$ & $5_{-t_{1}-t_{2}}$ & 0 & 3 & $3 \times\left(D, h_{u}\right)$ & + \\
\hline$\Sigma_{5^{(1)}}$ & $\overline{5}_{t_{1,2}+t_{3}}$ & 0 & -1 & $1 \times\left(d^{c^{\prime}}, \ell^{\prime}\right)$ & - \\
\hline$\Sigma_{5^{(2)}}$ & $\overline{5}_{t_{1,2}+t_{4}}$ & 0 & -1 & $1 \times\left(b^{c}, \ell_{\tau}\right)$ & - \\
\hline$\Sigma_{5^{(3)}}$ & $\overline{5}_{t_{1,2}+t_{5}}$ & 0 & -2 & $2 \times\left(\bar{D}, h_{d}^{\prime}\right)$ & - \\
\hline$\Sigma_{5^{(4)}}$ & $\overline{5}_{t_{3}+t_{4}}$ & 0 & -1 & $1 \times\left(d^{c}, \ell\right)$ & - \\
\hline$\Sigma_{5^{(5)}}$ & $\overline{5}_{t_{3}+t_{5}}$ & -1 & 0 & $1 \times h_{d}$ & + \\
\hline$\Sigma_{5^{(6)}}$ & $\overline{5}_{t_{4}+t_{5}}$ & 1 & -1 & $1 \times \bar{D}$ & - \\
\hline
\end{tabular}

Table 11. Field representation for $\mathcal{Z}_{2}$-case $C$ of $\mathcal{Z}_{2}$ monodromy.

$i=1,3,4$. Further we take $N=N_{9}=0$ and $N_{7}=-N_{5}=1$. From these arrangements, we get a complete tenplet $10^{(1)}=\left(Q, u^{c}, e^{c}\right)$ whose content we identify with the third generation. Because of the non-trivial flux, the remaining two tenplets decompose to pieces with different multiplicities. The $10^{(2)}$ in particular gives

$$
10^{(2)} \Rightarrow\left\{\begin{array}{l}
n_{(3,2)_{1 / 6}}-n_{(\overline{3}, 2)_{-1 / 6}}=1 \times Q \\
n_{(\overline{3}, 1)_{-2 / 3}}-n_{(3,1)_{2 / 3}}=2 \times u^{c} \\
n_{(1,1)_{1}}-n_{(1,1)_{-1}}=0
\end{array}\right.
$$

and the $10^{(3)}$ representation content is split according to

$$
10^{(2)} \Rightarrow\left\{\begin{array}{l}
n_{(3,2)_{1 / 6}}-n_{(\overline{3}, 2)_{-1 / 6}}=1 \times Q \\
n_{(\overline{3}, 1)_{-2 / 3}}-n_{(3,1)_{2 / 3}}=0 \\
n_{(1,1)_{1}}-n_{(1,1)_{-1}}=2 \times e^{c}
\end{array}\right.
$$

We choose $5^{(0)}=5_{h_{u}}$ to accommodate the up Higgs so that the coupling

$$
\mathcal{W} \supset 10^{(1)} 10^{(1)} 5^{(0)} \Rightarrow \lambda_{t} 10_{3} 10_{3} 5_{h_{u}}
$$

provides mass to the top-quark.

In the present case we take advantage of the possibility to realize the doublet-triplet splitting and simultaneously obtain a tree-level mass for the bottom quark, by assigning the down type Higgs to $\overline{5}^{(5)}=\overline{5}_{h_{d}}$ and making the following choice

$$
P=P_{5}=1, \quad P_{7}=0, \quad M^{a}=0, \quad M^{b}=2
$$

The resulting spectrum is shown in table 11 .

Then the relevant available coupling $10^{(1)} \overline{5}^{(2)} \overline{5}^{(5)}$ implies that the third generation can be assigned to $\overline{5}^{(2)}=\overline{5}_{3}$, thus

$$
\mathcal{W} \supset 10^{(1)} \overline{5}^{(2)} \overline{5}^{(5)} \Rightarrow \lambda_{b} 10_{3} \overline{5}_{3} \overline{5}_{h_{d}}
$$


However, in addition to the above the symmetries of the model allow also the couplings $10^{(1)} \overline{5}^{(1)} \overline{5}^{(6)}$ and $10^{(1)} \overline{5}^{(3)} \overline{5}^{(4)}$, involving dangerous 4-d proton decay operators.

A standard way to avoid such terms is to impose matter parity. In F-theory compactifications this is possible by engineering a $Z_{2}$-symmetry on both the Calabi-Yau fourfold $\mathcal{X}$ and the $G^{(4)}$ flux [45]. This symmetry is communicated to the matter curves so that the massless spectrum splits to positive and negative parity eigenstates. Provided a suitable $Z_{2}$ action is imposed on $\mathcal{X}, G^{(4)}$ pair, baryon violating and other unwanted terms can be eliminated as long as they do not conserve the induced $R$-parity. Choosing the $R$-parity of the various massless fields as in the last column of table 11, we can easily check that all the dangerous terms are eliminated.

We remark that this model has exactly the same chiral matter spectrum with the model presented in [22]. The requirement that this present version has an $\mathrm{SO}(10)$ origin has only led to an augmented Higgs sector by two more Higgs fiveplet pairs which can be massive by an appropriate coupling. In particular the following Yukawa couplings

$$
\mathcal{W} \supset\left\langle\theta_{15}\right\rangle \overline{5}^{(3)} 5^{(0)}+\left\langle\theta_{14}\right\rangle\left\langle\theta_{15}\right\rangle \overline{5}^{(6)} 5^{(0)}
$$

give heavy masses to the Higgs triplets. The model predicts a reasonable fermion mass hierarchy as discussed in [22]. Thus, we will not pursue it further here.

\section{$3.2 \quad \mathcal{Z}_{2} \times \mathcal{Z}_{2}$ models}

As noted above, compared to the previous $\left(\mathcal{Z}_{2}\right)$ case, now the number of curves is further reduced. Therefore, the possibilities of distributing matter on different curves are reduced and models arising in this context are eventually more constrained. The restrictions notwithstanding do not exclude a variety of models. We start by picking up the numbers

$$
M_{16}^{1}=3, \quad M_{16}^{3}=0, \quad M_{10}^{c}=0, \quad M_{10}^{b}=1, \quad N_{1}=0, \quad N_{2}=-1, \quad M=0, \quad P=0, \quad C=0
$$

Following the same procedure as above, we derive the SM spectrum shown in table 12. In addition, there are singlets $\theta_{i j}, i, j=1,2,3,4$ on the spectral curves defined in (2.3) as well as singlets $\theta_{i 5}$ from the $\mathbf{1 6}$ decompositions. The latter singlets in particular, appear on line bundles on the surface $S_{\mathrm{GUT}}$ and U(1) fluxes breaking the gauge group are affecting their chirality and multiplicities. Thus, we have

$$
\begin{aligned}
& \# \text { of } \theta_{15}-\# \text { of } \theta_{51}=M_{16}^{1}+(M-P)=3 \\
& \# \text { of } \theta_{35}-\# \text { of } \theta_{53}=M_{16}^{3}+P-C=0
\end{aligned}
$$

The results (3.20) tell us that there is an excess of $\theta_{15}$ over $\theta_{51}$ while possible $\theta_{35} / \theta_{53}$ arise only in pairs. Depending on the detailed geometrical properties of the compact space, these pairs can be either massive or massless. In the latter case they are expected to receive heavy masses and decouple.

The SO(10) Yukawa couplings are:

$$
\mathbf{1 6}_{t_{1}} \cdot \mathbf{1 6}_{t_{1}} \cdot \mathbf{1 0}_{t_{3}+t_{4}} \rightarrow 10^{(1)} 10^{(1)} 5^{0}+10^{(1)} \overline{5}^{(2)} \overline{5}^{(4)}+1_{t_{1}-t_{5}} \overline{5}^{(2)} 5^{(0)}
$$




\begin{tabular}{|c|c|c|c|c|c|}
\hline $\mathrm{SO}(10) \supset \mathrm{SU}(5)$ & $\mathrm{U}(1)_{i}$ & $N_{Y}$ & $\mathrm{U}(1)_{X}$ & SM reps & $R$-parity \\
\hline $16_{t_{1}} \supset 10^{(1)}$ & $t_{1,2}$ & 0 & 3 & $3 \times\left(Q, u^{c}, e^{c}\right)$ & - \\
\hline $16_{t_{3}} \supset 10^{(2)}$ & $t_{3}$ & -1 & 0 & $1 \times\left(u^{c}, \bar{e}^{c}\right)$ & - \\
\hline $10_{t_{3}+t_{4}} \supset 5^{(0)}$ & $-t_{1}-t_{2}$ & 0 & 1 & $1 \times\left(D, h_{u}\right)$ & + \\
\hline $10_{t_{1}+t_{4}} \supset 5^{(1)}$ & $-t_{1,2}-t_{3}$ & 0 & 0 & 0 & - \\
\hline $16_{t_{1}} \supset \overline{5}^{(2)}$ & $t_{1,2}+t_{5}$ & 0 & -3 & $3 \times\left(d^{c}, \ell\right)$ & - \\
\hline $16_{t_{3}} \supset 5^{(3)}$ & $-t_{3,4}-t_{5}$ & 1 & 0 & $1 \times h_{u}^{\prime}$ & - \\
\hline $10_{t_{3}+t_{4}} \supset \overline{5}^{(4)}$ & $t_{3}+t_{4}=2 t_{3}$ & -1 & -1 & $1 \times \bar{D}, 2 \times h_{d}$ & + \\
\hline $45 \supset 10^{(3)}$ & $t_{5}$ & 1 & 0 & $1 \times\left(\bar{u}^{c}, e^{c}\right)$ & - \\
\hline
\end{tabular}

Table 12. Field representation content under SU(5), and flux choices for the $\mathcal{Z}_{2} \times \mathcal{Z}_{2}$ case. Neutral singlet fields $\theta_{i j}$ are not shown in this table (see text).

Identifying $10^{(1)}=10_{M}, \overline{5}^{(2)}=\overline{5}_{M}, 5^{0}=5_{h_{u}}, \overline{5}^{(4)}=\overline{5}_{h_{d}}$ and $1_{t_{1}-t_{5}}=\theta_{15}$ we observe that all SM fermion masses arise from this coupling

$$
10_{M} 10_{M} 5_{h_{u}}, \quad 10_{M} \overline{5}_{M} \overline{5}_{h_{d}}, \quad \theta_{15} \overline{5}_{M} 5_{h_{u}}
$$

To avoid large Matter-Higgs mixing through the fiveplets, we take $\left\langle\theta_{15}\right\rangle=0$.

When the $\mathrm{U}(1)_{X, Y}$ fluxes are turned on, $\Sigma_{16_{t_{3}}}$ curve contains a $u^{c}$-type state descending from $16_{t_{3}}$ and a $\bar{e}^{c} \in \overline{16}_{t_{3}}$. Because $16_{t_{3}} \rightarrow 10^{(2)}+$ nothing, while due to the absence of $5^{(1)}$ (see table 12) the coupling

$$
\mathbf{1 6}_{t_{1}} \mathbf{1 6}_{t_{3}} \mathbf{1 0}_{t_{1}+t_{4}} \rightarrow\left\{10^{(1)}+\overline{5}^{(2)}+1_{t_{1}-t_{5}}\right\} \cdot\left\{10^{(2)}\right\} \cdot\{\text { nothing }\}
$$

does not provide any SU(5) invariant coupling.

Furthermore, the coupling

$$
\mathbf{4 5} \cdot \mathbf{1 6}_{t_{3}} \cdot \overline{\mathbf{1 6}}_{-t_{3}} \rightarrow 10^{(3)} \overline{10}^{(2)} \theta_{53}
$$

could in principle make extra matter massive, but the realization of such a coupling would require a $\theta_{53}$-vev from an additional $\theta_{35} / \theta_{53}$-pair provided that this pair is massless. The coupling

$$
\theta_{13}^{2} 5^{(0)} \overline{5}^{(4)} \rightarrow\left\langle\theta_{13}^{2}\right\rangle D \bar{D}
$$

makes extra triplets massive, whilst the coupling

$$
\left(\theta_{13}^{2} \overline{5}^{(0)}+\theta_{53} 5^{(3)}\right) \overline{5}^{(4)}
$$

could make massive one linear combination of $h_{u}$ and one $h_{d}$ doublets.

\subsubsection{Minimal variation}

The above minimal version appears to be a promising scenario provided there is a way to make extraneous matter massive. One of the possible issues here is that it requires a singlet vev which appears only in pairs $\theta_{53} / \theta_{35}$ and without knowing the detailed geometry we 


\begin{tabular}{|c|c|c|c|c|}
\hline $\mathrm{SO}(10) \supset \mathrm{SU}(5)$ & $\mathrm{U}(1)_{i}$ & $N_{Y}$ & $\mathrm{U}(1)_{X}$ & $\mathrm{SM}$ reps \\
\hline $16_{t_{1}} \supset 10^{(1)}$ & $t_{1,2}$ & 0 & 3 & $3 \times\left(Q, u^{c}, e^{c}\right)$ \\
\hline$\overline{16}_{-t_{3}} \supset \overline{10}^{(2)}$ & $-t_{3}$ & -1 & -1 & $1 \times \bar{Q}^{\prime}, 2 \times \bar{e}^{c^{\prime}}$ \\
\hline $10_{t_{3}+t_{4}} \supset 5^{(0)}$ & $-t_{1}-t_{2}$ & 0 & 1 & $1 \times\left(D, h_{u}\right)$ \\
\hline $10_{t_{1}+t_{3}} \supset 5^{(1)}$ & $-t_{1,2}-t_{3}$ & 0 & 0 & 0 \\
\hline $16_{t_{1}} \supset \overline{5}^{(2)}$ & $t_{1,2}+t_{5}$ & 0 & -3 & $3 \times\left(d^{c}, \ell\right)$ \\
\hline $16_{t_{3}} \supset 5^{(3)}$ & $-t_{3,4}-t_{5}$ & 1 & 0 & $1 \times h_{u}^{\prime}$ \\
\hline $10_{t_{3}+t_{4}} \supset \overline{5}^{(4)}$ & $t_{3}+t_{4}=2 t_{3}$ & -1 & -1 & $1 \times \bar{D}, 2 \times h_{d}$ \\
\hline $45 \supset 10^{(3)}$ & $t_{5}$ & 1 & 1 & $1 \times Q^{\prime}, 2 \times e^{c^{\prime}}$ \\
\hline $16_{t_{1}} \supset 1^{(1)}$ & $t_{1}-t_{5}$ & 0 & 3 & $3 \times \theta_{15}$ \\
\hline$\overline{16}_{-t_{3}} \supset 1^{(3)}$ & $-t_{3}+t_{5}$ & -1 & -1 & $2 \times \theta_{53}$ \\
\hline
\end{tabular}

Table 13. A modified field representation content under $\mathrm{SO}(10) \supset \mathrm{SU}(5)$, for the $\mathcal{Z}_{2} \times \mathcal{Z}_{2}$ case. Singlets arising from 16's are also included. (There are additional singlets from SU(4) spectral cover not include here, see text.)

cannot claim whether it is massless or not. We could replace this by other combinations, like $\theta_{54} \theta_{43}$ etc. Another way is to modify some of the flux parameters.

Thus, in addition to the non-zero integers in (3.19), let us assume also non-zero values for

$$
M_{16}^{3}=-1, \quad M_{10}^{c}=C=+1
$$

Then, the number of $5^{(3)}$ 's does not change but the number of singlets becomes

$$
\text { \# of } \theta_{35}-\# \text { of } \theta_{53}=M_{16}^{3}+P-C=-2
$$

thus, we have two copies of $\theta_{53}$. There are modifications on the exotic spectrum too. In particular

$$
10^{(2)} \rightarrow 1 \times \bar{Q}^{\prime}+2 \bar{e}^{c}, \quad 10^{(3)} \rightarrow 1 \times Q^{\prime}+2 e^{c}
$$

The modified massless spectrum is given in table 13 .

We discuss now the modifications induced on Yukawa couplings. Because $\theta_{53}$ is now present in the spectrum, the coupling (3.23) is now suitable to provide a mass to a pair $h_{u}^{\prime} h_{d}^{\prime}$. However, the chiralities of the representations involved in (3.21) are reversed. To make extra matter massive we need a coupling of the form

$$
\overline{10}_{-t_{3}}^{(2)} 10_{t_{5}} \Phi_{t_{3}-t_{5}}
$$

where $\Phi_{t_{3}-t_{5}}$ an effective singlet to compensate for a pissible absence of a massless $\theta_{35}$. Indeed, we could have for example

$$
\Phi_{t_{3}-t_{5}}=\theta_{31} \theta_{15}
$$

The $\mathrm{SO}(10)$ origin of $(3.25)$ is

$$
\overline{\mathbf{1 6}}_{-t_{3}} \cdot \mathbf{4 5} \cdot \mathbf{1 6}_{t_{1}} \theta_{31}
$$


Notice that $\theta_{31}$ is a singlet even at the $\mathrm{SO}(10)$ level, since its origin is from the spectral cover SU(4) adjoint

$$
15 \rightarrow \sum_{i \neq j}^{4} \mathbf{1}_{t_{i}-t_{j}}+3 \times \mathbf{1}_{0} \rightarrow \theta_{i j}+3 \times \theta_{0}
$$

Before closing this section, we take the opportunity to add a few more comments with regard to the singlet fields: we have already seen that $\mathrm{U}(1)_{X}$ flux leaves an excess of $\theta_{53}$ fields over $\theta_{35}$, the specific number of them being determined in terms of the integers related to the appropriate flux. In general there may also be vector-like pairs $\theta_{53}+\theta_{35}$, massless or massive depending on the specific internal geometry. These singlets appear on line bundles on the surface $S_{\mathrm{GUT}}$ and U(1) fluxes breaking the gauge group are affecting their chirality and multiplicities in the way described above. However, the properties of singlets that do not localize on line bundles on $S_{\mathrm{GUT}}$ are not always known. In general, it is observed that chirality is also present in the singlet field spectrum as long as these singlet fields reside on matter curves on $S_{\mathrm{GUT}}$. If a similar flux mechanism is operative at a preceding symmetry level, it could also eliminate some of $\theta_{i j}, i, j=1,2,3,4$. As we have already mentioned at the $\mathrm{SO}(10)$ level $\theta_{13}$ and $\theta_{31}$ are $\mathrm{SO}(10)$ singlets and therefore are found on line bundles extending normal to $S_{\mathrm{GUT}}$ and away from the local point on $S_{\mathrm{GUT}}$. Although at our present level of understanding we do not know the details of the global geometry and the breaking mechanism away from $S_{\mathrm{GUT}}$ we can possibly deduce such properties [24] near $S_{\mathrm{GUT}}$ from the spectral cover. In the above analysis we assume the existence of both fields since as we have seen both are needed to give mass to extraneous matter fields, otherwise one would need a contrived way to get rid of these states.

\section{$3.3 \quad \mathcal{Z}_{3}$ models}

Finally, in this section we present two characteristic examples of effective models when we quotient the theory with a $\mathcal{Z}_{3}$ monodromy.

\subsubsection{First example}

We make the following choice

$$
M_{16}^{1}=4, \quad M_{16}^{2}=-1, \quad M=-1, \quad P=-1, \quad C=3, \quad M_{10}=2 .
$$

Then, the required combinations take the values

$$
M_{10}+M=+1, \quad-M_{10}+M=-3, \quad-M_{16}^{1}+(M-P+C)=-1, \quad-M_{16}^{2}+P=0 .
$$

The massless spectrum is presented in table 14. Remarkably, this model is the $\mathrm{SO}(10)$ upgrade of the $3+1+1+1$ example given in [21]. Here, in addition we are also able to obtain the exact singlet field spectrum whose properties are determined by the $\mathrm{U}(1)_{X}$ flux. Notice that the singlets $\theta_{i j}$ with $i, j=1,2,3$ because of the identification $t_{1}=t_{2}=t_{3}$ under $\mathcal{Z}_{3}$, carry no charge and therefore they do not couple to the fluxes. Further, the multiplicities of $\theta_{i 4}, \theta_{4 i}$ are not determined by the $\mathrm{U}(1)_{X, Y}$ fluxes assumed here, hence they are treated as free parameters. 


\begin{tabular}{|c|c|c|c|c|}
\hline $\mathrm{SO}(10) \supset \mathrm{SU}(5)$ & $N_{Y}$-flux & $M_{X}$ & Matter & $R$-parity \\
\hline $16_{t_{i}} \supset 10_{t_{i}}^{(1)}=10_{M}$ & +1 & 4 & $4 Q+3 u^{c}+5 e^{c}$ & - \\
\hline$\overline{16}_{-t_{4}} \supset \overline{10}_{-t_{4}}^{(2)}=\overline{10}_{x}$ & -1 & -1 & $1 \bar{Q}+2 \bar{e}^{c}$ & - \\
\hline $10_{-2 t_{i}} \supset 5_{-2 t_{i}}^{(0)}=5_{h_{u}}$ & 0 & +1 & $h_{u}+D$ & + \\
\hline $10_{t_{i}+t_{4}} \supset \overline{5}_{t_{i}+t_{4}}^{(1)}=\overline{5}_{M}$ & 0 & -3 & $3\left(\ell+d^{c}\right)$ & - \\
\hline $16_{t_{i}} \supset \overline{5}_{t_{i}+t_{5}}^{(2)}$ & +1 & -1 & $\bar{D}$ & - \\
\hline $16_{t_{4}} \supset \overline{5}_{t_{4}+t_{5}}^{(3)}$ & -1 & 0 & $h_{d}$ & + \\
\hline $16_{t_{i}} \supset 1_{t_{i}-t_{5}}$ & +1 & 4 & $7 \times \theta_{15}$ & - \\
\hline$\overline{16}_{-t_{4}} \supset \overline{1}_{t_{5}-t_{4}}$ & -1 & -1 & $2 \times \theta_{54}$ & - \\
\hline $45 \supset 10_{t_{5}}^{(3)}$ & 0 & 0 & empty & - \\
\hline
\end{tabular}

Table 14. Field representation content under SU(5), their homology class and flux restrictions under $\mathrm{U}(1)_{Y}$ for the first example of the $\mathcal{Z}_{3}$ case.

SO(10) origin of couplings. The top Yukawa can be obtained from

$$
\mathbf{1 6}_{t_{i}} \mathbf{1 6}_{t_{i}} \mathbf{1 0}_{-2 t_{i}} \rightarrow 10_{M} 10_{M} 5_{h_{u}}+10_{M} \overline{5}_{t_{i}+t_{5}}^{(2)} \overline{5}_{M}+\theta_{15} \overline{5}_{t_{i}+t_{5}}^{(2)} 5_{h_{u}}
$$

The first term is indeed the up-quark mass matrix, while the third one provides mass to the extra triplet pair through a non-zero vev $M_{D}=\left\langle\theta_{15}\right\rangle$ :

$$
\left\langle\theta_{15}\right\rangle \overline{5}_{t_{i}+t_{5}}^{(2)} 5_{h_{u}} \rightarrow M_{D} \bar{D} D
$$

Notice that according to our choices, $10_{-2 t_{i}}$ contributes $M_{10}^{b}-4$ multiplets of $5_{h_{u}}$ 's, thus we need to assume $M_{10}^{b}>4$. Since $M_{10}^{b}-M_{10}^{a}=2$, it turns out that $M_{10}^{a}>2$. Taking $M_{10}^{a}=3$, then $10_{2 t_{1}}$ gives $M_{10}^{a}-3=0$ of $\overline{5}_{2 t_{1}}$ 's and the coupling

$$
\mathbf{1 6}_{t_{i}} \mathbf{1 6}_{t_{4}} \mathbf{1 0}_{2 t_{i}} \rightarrow 10_{M} \overline{5}_{t_{4}+t_{5}}^{(3)} \bar{\not}_{2 t_{1}}
$$

is not realized because $\overline{5}_{2 t_{1}} \notin 10_{2 t_{1}}$. The $\mathrm{SO}(10)$ origin of the bottom coupling is

$$
\mathbf{1 6}_{t_{i}} \mathbf{1 0}_{t_{j}+t_{4}} \mathbf{1 6}_{t_{4}} \theta_{k 4} \rightarrow 10_{M} \overline{5}_{M} \overline{5}_{h_{d}} \theta_{14}, \quad\{i, j, k\}=\{1,2,3\}
$$

A Higgs mixing term would require two SU(5) singlets obtained from

$$
\theta_{14} \mathbf{1 6}_{t_{i}} \mathbf{1 6}_{t_{4}} \mathbf{1 0}_{-2 t_{j}} \rightarrow \theta_{14} \theta_{15} \overline{5}_{h_{d}} 5_{h_{u}}
$$

From the bottom mass term, we infer that $\left\langle\theta_{14}\right\rangle$ should be large enough. Similarly the triplet mass requires also a non-zero $\left\langle\theta_{15}\right\rangle$. These requirements induce unacceptably large Higgs mixing. However, it is possible to keep triplets light without disturbing the RGE running [46]-[51]. In this case we could assume a small $\left\langle\theta_{15}\right\rangle$ vev. Alternatively, we could impose matter parity to eliminate this term. We discuss this issue in conjunction with the requirements to avoid possible proton decay operators. Notice first that $h_{u}, h_{d}$ reside on different matter curves, while the triplet $\overline{5}_{h_{d}}$ has been washed away by flux, therefore, the relevant tree-level graph mediated by the triplet cannot be generated. Even if massive 


\begin{tabular}{|c|c|c|c|c|}
\hline $\mathrm{SU}(5)$ & $N_{Y^{-}}$flux & $M_{X}$ & Matter & $R$-parity \\
\hline $10_{t_{i}}^{(1)}=10_{M}$ & 0 & 3 & $3\left(Q+u^{c}+e^{c}\right)$ & + \\
\hline $5_{-2 t_{i}}^{(0)}=5_{h_{u}}$ & 0 & +1 & $h_{u}+D$ & + \\
\hline$\overline{5}_{t_{i}+t_{4}}^{(1)}=\overline{5}_{\bar{D}}$ & +1 & -1 & $\bar{D}$ & \pm \\
\hline$\overline{5}_{t_{i}+t_{5}}^{(2)}=\overline{5}_{h_{d}}$ & -1 & 0 & $h_{d}$ & \pm \\
\hline$\overline{5}_{t_{4}+t_{5}}^{(3)}=\overline{5}_{M}$ & 0 & -3 & $3\left(d^{c}, \ell\right)$ & - \\
\hline$\left[\theta_{14} \cdot \overline{5}^{1}\right]$ & & & & + \\
\hline$\left[\theta_{15} \cdot \overline{5}^{2}\right]$ & & & & - \\
\hline$\left[\theta_{15} \cdot \theta_{14}\right]$ & & & & - \\
\hline
\end{tabular}

Table 15. Field representation content under SU(5), their homology class and flux restrictions under $\mathrm{U}(1)_{Y}$ for the second example of the $\mathcal{Z}_{3}$ case. Last column shows the $R$-parity assignment used to eliminate unwanted operators.

KK-modes of the above states are considered, they could not form a direct term. Therefore, proton decay graphs are expected to be suppressed.

Notice also that the operator

$$
1 \mathbf{6}_{t_{i}} \mathbf{1 6}_{t_{i}} \mathbf{1 6}_{t_{i}} \mathbf{1 0}_{t_{i}+t_{4}} \rightarrow 10_{t_{i}} 10_{t_{i}} 10_{t_{i}} 5_{t_{i}+t_{4}}
$$

would require a non-zero $\theta_{5 i}$-vev which does not exist.

Further, we see that the $\mathrm{SO}(10)$ coupling

$$
\theta_{14} \mathbf{1 0}-2 t_{1} \mathbf{1 0}_{t_{1}+t_{4}} \rightarrow\left\langle\theta_{14}\right\rangle 5_{h_{u}} \overline{5}_{M}
$$

would imply unacceptable mixing among color extra triplets and ordinary matter.

As illustrated in [45], provided a suitable $Z_{2}$-symmetry is imposed on $\mathcal{X}, G^{(4)}$ pair, baryon violating and other unwanted terms can be eliminated if they do not conserve the induced $R$-parity. Choosing the $R$-parity of the various massless fields as in the last column of table 14, we can easily check that all the dangerous terms are eliminated. Notice also that the mere existence of the bottom Yukawa coupling (3.26), requires that the $\mathrm{SO}(10)$ singlet $\theta_{14}$ should be assigned with positive $(+) R$-parity. This makes impossible the existence of the Higgs mixing term (3.27), thus the vev of $\theta_{15}$ can be chosen at will.

\subsubsection{Second example}

For the second Ansatz of the $\mathcal{Z}_{3}$ case, we choose $N_{x}=-N_{y}=1, M_{16}^{1}=3, M_{10}=1$, $P=-3$ and $M=C=0$, to obtain a rather interesting model with the spectrum presented in table 15. There is also extraneous matter coming in pairs $\bar{e}^{c} e^{c}, \bar{u}^{c} u^{c}$ from the $10^{2,3}$ representations which can be massive by an appropriate coupling $\sim\langle X\rangle\left(\bar{e}^{c} e^{c}+\bar{u}^{c} u^{c}\right)$.

The couplings providing with masses the charged fermions and the Higgs triplets are

$$
10^{1} 10^{1} 5^{0}+10^{1} \overline{5}^{3} \overline{5}^{2} \theta_{15}+5^{0} \overline{5}^{1} \theta_{14} \rightarrow 10_{M} 10_{M} 5_{h_{u}}+10_{M} \overline{5}_{M} \overline{5}_{h_{d}}+\left\langle\theta_{14}\right\rangle 5_{h_{u}} \overline{5}_{\bar{D}}
$$

Notice that the bottom mass originates from a fourth order NR-term, thus the corresponding singlet vev should be substantially large $\left\langle\theta_{15}\right\rangle \gtrsim 10^{-1} M_{S}$, with $M_{S}$ being the 
GUT-scale. Consequently, the coupling $5^{0} \overline{5}^{2} \theta_{15}$ involving the same vev $\left\langle\theta_{15}\right\rangle$ should be avoided to protect Higgs doublets from receiving a large mass. In addition dimension four and five proton decay operators $10_{M} \overline{5}_{M} \overline{5}_{M} \theta_{14} \theta_{15}$ and $10_{M} 10_{M} 10_{M} \overline{5}_{M}$ allowed by gauge symmetry, should be eliminated. To this end we again appeal to the matter parity which for the present model is chosen according to the last column of table 15 . We observe that the above requirements fix only the product $\theta_{14} \overline{5}^{1}$ to positive, and $\theta_{15} \overline{5}^{2}, \theta_{14} \theta_{15}$ to negative $R$-parity. This choice is also compatible with the neutrinos. Left handed neutrino components living in $\ell=(\nu, e)$ of $\overline{5}_{M}$ should be paired up with right-handed components $\nu^{c}$, which at the SM gauge symmetry level should generate mass terms of the form

$$
\mathcal{W}_{\nu} \sim \lambda_{\nu} h_{u} \ell \nu^{c}+M \nu^{c} \nu^{c}
$$

If we assign matter parity $(+)$ to the $\nu^{c}$ state, the first term which gives a Dirac mass could be contained in a non-renormalizable coupling of the form $\overline{5}_{M} 5_{h_{u}} \nu^{c}\left\langle\theta_{14} \theta_{15}\right\rangle$. The role of the right-handed neutrino could be played by the zero modes $\theta_{i i}$ which are neutral under the U(1) factors. Alternatively, the right-handed neutrinos can be the Kaluza-Klein modes as in [52] and its F-theory extension [53]. We remind that we have left an ambiguity in the determination of matter parities of the corresponding states in table 15. In the next section, we will see how this is fixed from the intrinsic geometry considerations.

\section{Matter parity from geometry}

In several cases of the models above, in order to prevent proton decay operators we have appealed to matter parity that might arise from the internal geometry and the fluxes. The implementation of this idea requires a thorough study of the manifold and the flux properties along the lines of the discussion started in [45]. ${ }^{4}$

A simpler bottom-up approach to incarnate such symmetries in a local model could be described as follows. We consider the GUT divisor $S_{\mathrm{GUT}}$ which locally is covered by open patches $U_{a} \in S_{\mathrm{GUT}}$. We focus on a single trivialization patch and take $s$ to be the coordinate along the fiber. Here we will relax other possible constraints [45] and simply demand that under the required geometric transformation the spectral cover equation should remain invariant up to an overall phase. To this end consider the transformation $\sigma$ where $s, b_{k}$ are mapped according to

$$
s(\sigma(p))=s(p) e^{i \phi}, \quad b_{k}(\sigma(p))=b_{k}(p) e^{i(\xi-(6-k) \phi)}
$$

Then each term in the spectral cover equation transforms the same way

$$
b_{k} s^{5-k} \rightarrow e^{i(\xi-\phi)} b_{k} s^{5-k}
$$

This invariance allows two different ways to communicate a $Z_{2}$ symmetry to $S_{\mathrm{GUT}}$ :

- For $\phi=0$

$$
s \rightarrow s, \quad b_{k} \rightarrow b_{k} e^{i \xi}
$$

\footnotetext{
${ }^{4}$ For other scenarios implementing such symmetries in F-theory models see [23, 51]. The importance of deriving such symmetries of string origin has also been discussed recently in [54] and in the context of Gepner model in [55].
} 
- For a $Z_{N}$ symmetry we take

$$
\phi=\frac{2 \pi}{N}
$$

thus, for $N=2$, we have $\phi=\pi$ and

$$
s \rightarrow-s, \quad b_{k} \rightarrow(-1)^{k} e^{i \xi} b_{k}
$$

The geometric $Z_{2}$ symmetry which preserves the spectral cover equation can be associated to a transformation of the complex coordinates which can also induce a $Z_{2}$ transformation on spinors [45]. In most of the phenomenological models a matter parity is identified as a $Z_{2}$ symmetry in the superpotential of chiral matter and Higgs fields. We can construct a matter parity based on the above considerations by extending the above $Z_{2}$ symmetry to the line bundles associated to the matter and Higgs representations of $\mathrm{SU}(5)$. To do this, we need to know the particular way that these properties are induced to the corresponding wavefunctions. We recall here that a zero-mode chiral multiplet is identified as a section of a line bundle on a matter curve and the corresponding wavefunction is determined as a holomorphic section on the covering matter curve. Therefore, in the local picture we only need to introduce in a consistent way a $Z_{2}$ symmetry transformation on these line bundles to associate a matter parity to the zero modes. We should remark here that in several cases of the subsequent explorations the resulting matter parity can differ from the conventional one. Yet, we will see that these cases can lead to viable effective models.

\subsection{A $Z_{2}$ parity for the $\mathcal{Z}_{2} \times \mathcal{Z}_{2}$ monodromy}

From the previous discussion, we infer that a reasonable way to communicate consistently this parity to the various states residing on the matter curves is through the coefficients $a_{n}$ and their relations implied by the splitting of the spectral cover. Consider for example the case of $\mathrm{SU}(5)$ for $\mathcal{Z}_{2} \times \mathcal{Z}_{2}$. Then the $b_{k}$ 's are given in terms of $a_{m}$ 's by relations of the form

$$
b_{k}=\sum a_{l} a_{m} a_{n}, \quad l+m+n=N-k
$$

where $N=17$. If $a_{n}$ transform as

$$
a_{n} \rightarrow a_{n} e^{i(\zeta-n \phi)}
$$

then

$$
b_{k} \propto a_{l} a_{m} a_{n} \rightarrow a_{l} a_{m} a_{n} e^{3 \zeta-(N-k) \phi}
$$

and for $\phi=\pi$ the phase of $b_{k}$ is

$$
(-1)^{k+1} e^{i 3 \zeta}
$$

which is consistent with (4.1) for example if $\xi=\pi, \zeta=0$, thus

$$
a_{n}(\sigma(p))=a_{n}(p) e^{-i n \pi}
$$

We can in principle use several ways to correlate these phases to the particular properties of the line bundles and the wavefunctions associated to them, which we now discuss. We 


\begin{tabular}{|c|c|c|c|}
\hline $\mathrm{SU}(5)$ & Equation & Matter & $R$-parity \\
\hline $10_{t_{i}}^{(1)}=10_{M}$ & $a_{1}$ & $4 Q+3 u^{c}+5 e^{c}$ & - \\
\hline$\overline{10}_{-t_{4}}^{(2)}=\overline{10}_{x}$ & $a_{5}$ & $1 \bar{Q}+2 \bar{e}^{c}$ & - \\
\hline $5_{-2 t_{i}}^{(0)}=5_{h_{u}}$ & $a_{1} a_{6} a_{8}+\ldots$ & $h_{u}+D$ & + \\
\hline$\overline{5}_{t_{i}+t_{4}}^{(1)}=\overline{5}_{M}$ & $a_{1} a_{6}+a_{2} a_{5}$ & $3\left(\ell+d^{c}\right)$ & - \\
\hline$\overline{5}_{t_{i}+t_{5}}^{(2)}$ & $a_{2} a_{7}+a_{1} a_{8}$ & $\bar{D}$ & - \\
\hline$\overline{5}_{t_{4}+t_{5}}^{(3)}$ & $a_{6} a_{7}+a_{5} a_{8}$ & $h_{d}$ & + \\
\hline
\end{tabular}

Table 16. Field representation content under SU(5), the defining equations and matter content and parity for the $\mathcal{Z}_{3}$ case (the indices $i, j, k$ take the values $1,2,3$ ).

\begin{tabular}{|l|ccccccc|}
\hline Representation & $10^{(1)}$ & $10^{(2)}$ & $10^{(3)}$ & $5^{(0)}$ & $\overline{5}^{(1)}$ & $\overline{5}^{(2)}$ & $\overline{5}^{(3)}$ \\
Phase $n \pi$ & $\pi$ & $5 \pi$ & $7 \pi$ & $15 \pi$ & $7 \pi$ & $9 \pi$ & $13 \pi$ \\
$e^{n \bmod (13) i \pi}$ & - & - & - & + & - & - & + \\
\hline
\end{tabular}

Table 17. 'Geometric' origin of matter parity for the $\mathcal{Z}_{3}$ model A.

can rely for example on the fact that the bundles are associated to particular products of the coefficients $a_{n}$ being sections of the sums of the latter. The $5^{(0)}$ fiveplet for example has a defining equation involving the combinations $a_{6} a_{7}+a_{5} a_{8}$. Under the preceding transformation, it gives an overall phase

$$
5^{(0)} \sim a_{6} a_{7} \rightarrow(-1)^{13}=-1
$$

If we associate these phases to the $R$-parity then the representations transform according to:

$$
10^{(i)}(-), 5^{(0)}(-), 5^{(1)}(+), 5^{(2)}(-), 5^{(3)}(+), 5^{(4)}(-)
$$

However, this is not the parity used in table 12 thus either another identification of the matter spectrum should be used or a more sophisticated parity construction should be associated to the matter curves.

\subsection{The case of $\mathcal{Z}_{3}$ models}

- For an alternative way to define the parity operation we consider the model $A$ of the $\mathcal{Z}_{3}$ monodromy discussed previously, (originally given in [21]). The massless spectrum is presented in table 16. Following the same procedure to each representation of the model we can associate a phase shown as in the second line of table 17 . The matter parity obtained by the operation $(\bmod 13)$ is given in the third line of table 17 and is in accordance with that one used in our preceding discussion of the $\mathcal{Z}_{3}$ model (see table 16). Although such an operation looks farfetched and a rather contrived attempt to match the parities imposed by hand on the various models discussed, it still paves the way to consider alternative - and possibly more realistic - methods to construct a parity consistent with the local geometry. 
- Next, we discuss another case which associates successfully matter parities to matter curves in a simpler way. Indeed, a rather simple assignment works for the model $B$ of the $\mathcal{Z}_{3}$ case. Assume that all $a_{l}$ 's transform according to

$$
a_{l} \rightarrow(-)^{l+1} a_{l}
$$

Using the defining equations (2.24) we find the following assignment

$$
10^{1}(+), 5^{0}(+), \overline{5}^{1}(-), \overline{5}^{2}(-), \overline{5}^{3}(-)
$$

where the matter parity is shown in the brackets next to the representations. This is compatible with the last column of table 15 , provided we choose $\theta_{14}=(-), \theta_{15}=(+)$.

\subsection{Matter parity for the $\mathcal{Z}_{2}$ model $C$}

For the model $C$ of the $\mathcal{Z}_{2}$ case, we can construct a parity demanding that $a_{i}$ 's transform as follows

$$
a_{n} \rightarrow a_{n}, \quad \text { for } n=6,7, \quad a_{m} \rightarrow-a_{m}, \quad \text { for } m \neq 6,7 .
$$

We can check from (B.6) that all $b_{k}$ transform the same way, $b_{k} \rightarrow-b_{k}$ and for $s \rightarrow s$ the spectral cover equation picks up only an overall minus sign. From equations (2.14) and (2.16) we observe that $5^{(0)}, 5^{(5)}$ and $10^{(3)}$ obtain a positive R-parity while the remaining representations acquire negative R-parity. We observe that this matches the parity imposed on the model of table 11 except for the $10^{(3)}$ representation which changes sign. This would imply minor modifications of the model, the most important being on the fermion mass textures. Indeed, recalling that $10^{(3)}$ accommodates the lightest generation [22], we can see that by choosing the parities of the singlets $\theta_{14}=(-)$ and $\theta_{43}=(+)$, we can generate realistic fermion mass textures with affordable zero-entries. Notice that the dangerous dimension four operators $10_{M}^{(3)} \overline{5}_{M}^{(i)} 5_{M}^{(j)} \theta_{5 k}$, although now could be allowed, they would require singlet vevs $\left\langle\theta_{5 k}\right\rangle$ which can be taken to be zero.

\subsection{A $Z_{2}$ matter parity for a model with $2+3$ spectral cover split}

We now apply the idea of matter parity to a toy model with $2+3$ splitting. This model was first analyzed in refs $[17,21]$, however, we will see that in the present approach we are forced to introduce a different R-parity. The spectral cover equation is written as

$$
P_{5}=\left(a_{1}+a_{2} s+a_{3} s^{2}+a_{4} s^{3}\right)\left(a_{5}+a_{6} s+a_{7} s^{2}\right)
$$

This leads to the identifications $t_{1,2,3}=t_{a}$ and $t_{4,5}=t_{b}$, with the trace condition now reading $3 t_{a}+2 t_{b}=0$. The equations connecting $b_{k}$ 's with $a_{i}$ 's are of the form $b_{k} \sim$ $\sum_{n} a_{n} a_{11-n-k}$ for appropriate values of $n$, in particular

$$
\begin{aligned}
& b_{0}=a_{4} a_{7} \\
& b_{1}=a_{3} a_{7}+a_{4} a_{6} \\
& b_{2}=a_{2} a_{7}+a_{3} a_{6}+a_{4} a_{5} \\
& b_{3}=a_{1} a_{7}+a_{2} a_{6}+a_{3} a_{5} \\
& b_{4}=a_{1} a_{6}+a_{2} a_{5} \\
& b_{5}=a_{1} a_{5}
\end{aligned}
$$




\begin{tabular}{|c|c|c|c|c|c|c|}
\hline $\mathrm{SU}(5)$ & Equation & Homology & $N_{Y}$ & $M_{X}$ & Matter & $R$ \\
\hline$\overline{10}_{-t_{a}}^{(1)}=\overline{10}_{x}$ & $a_{1}$ & $\eta-3 c_{1}-\chi$ & -1 & -1 & $\left(\bar{Q}, 2 \bar{e}^{c}\right)$ & - \\
\hline $10_{t_{b}}^{(2)}=10_{M}$ & $a_{5}$ & $-2 c_{1}+\chi$ & 1 & 4 & $\left(4 Q, 3 u^{c}, 5 e^{c}\right)$ & - \\
\hline$\overline{5}_{t_{a}+t_{b}}^{(0)}=\overline{5}_{h_{d}}$ & $a_{1}^{2} a_{7}+a_{1} a_{2} a_{6}+\cdots$ & $2 \eta-6 c_{1}-\chi$ & -1 & 0 & $h_{d}$ & - \\
\hline$\overline{5}_{2 t_{a}}^{(1)}=5_{M}$ & $a_{1} a_{7}+a_{2} a_{6}$ & $\eta-3 c_{1}$ & 0 & -3 & $3\left(d^{c}, \ell\right)$ & + \\
\hline $5_{-2 t_{b}}^{(2)}=5_{h_{u}}$ & $a_{6}$ & $-c_{1}+\chi$ & 1 & 0 & $h_{u}$ & + \\
\hline
\end{tabular}

Table 18. Field representation content under SU(5), their homology class and flux restrictions under $\mathrm{U}(1)_{Y}$ for the $\mathcal{Z}_{3} \times \mathcal{Z}_{2}$ case (the indices take the values $a=1,2,3, b=4,5$ ).

We further demand the following transformations:

$$
a_{m}(\sigma(p))=a_{m}(p) e^{i m \pi}=(-)^{m} a_{m}(p), \quad \text { for } m=1, \ldots, 7
$$

The constraint $b_{1}=a_{3} a_{7}+a_{4} a_{6}=0$ can be solved by $a_{4}=-\lambda a_{7}$ and $a_{3}=\lambda a_{6}$. Repeating the steps as in the previous cases, we determine the homologies and flux restrictions of the spectrum given in table 18. Assuming the simplest scenario, we associate the matter parity with the phase of the defining equation of the second column. Using (4.2) for $a_{n}$ 's above, we obtain the parities of the last column. Notice that the latter does not coincide with the parity chosen in $[17,21]$.

Note, that there are also $\mathrm{SU}(5)$ singlets $\theta_{i j}$ obtained from the $24 \in \mathrm{SU}(5)_{\perp}$ residing on curves extended away from $S_{\mathrm{GUT}}$. After the monodromy identifications they can be organized into two categories. Those carrying $\mathrm{U}(1)_{i}$-charges are denoted with $\theta_{a b}, \theta_{b a}$ and the 'neutral' ones $\theta_{a a}, \theta_{b b}$ which can be identified with the neutrinos. Since we do not know the global geometry we will treat their parities as free parameters.

We distribute the matter and Higgs fields over the curves as follows

$$
10_{t_{b}}=10_{M}, \quad \overline{5}_{t_{a}+t_{b}}=\overline{5}_{h_{d}}, \quad 5_{-2 t_{b}}=5_{h_{u}}, \quad \overline{5}_{2 t_{a}}=\overline{5}_{M}, \quad \overline{10}_{-2 t_{a}}=\overline{10}
$$

while we determine the multiplicities by choosing $N=1$ and the $M_{X}$ 's as shown in tha table 18 .

The allowed tree-level couplings with non-trivial SU(5) representations are

$$
\mathcal{W}_{\text {tree }}=\lambda_{u} 10_{M} 10_{M} \overline{5}_{h_{u}}+\lambda_{d} 10_{M} \overline{5}_{M} \overline{5}_{h_{d}}+\lambda_{x} \overline{10}_{x} 10_{M} \theta_{a b}
$$

The first two provide masses to quarks and charged leptons. The third term survives by assuming positive matter parity for $\theta_{a b}$, while a non-zero vev gives masses to the exotic matter.

The dangerous dimension four and five operators (namely $10_{M} \overline{5}_{M} \overline{5}_{M}$ and $\left.10_{M} 10_{M} 10_{M} \overline{5}_{M}\right)$ inducing proton decay are eliminated under the combined action of parity and U(1) symmetry. Notice also that the Higgs mixing term $5_{h_{u}} \overline{5}_{h_{d}} \theta_{a b}$ is prevented by R-parity. 


\section{Conclusions}

In the present work we have analyzed several aspects of the local F-theory GUTs associated to $\mathrm{SO}(10)$ and $\mathrm{SU}(5)$ singularities of the internal geometry. We have considered the analysis in a spectral cover context where these symmetries are incorporated in $E_{8}$ which is assumed to be the maximum singularity of the internal manifold. We have investigated several implications on the derived models of all possible monodromies among the $\mathrm{U}(1)$ symmetries emerging from the $\mathrm{SU}(4)_{\perp}$ and the $\mathrm{SU}(5)_{\perp}$ spectral covers corresponding to $\mathrm{SO}(10)$ and $\mathrm{SU}(5)$ gauge symmetries respectively.

In particular, we have investigated systematically the landscape of effective models with $\mathrm{SO}(10)$ and $\mathrm{SU}(5)$ gauge symmetries emerging under the various cases of monodromies among abelian factors embedded in the $\mathrm{SU}(4)_{\perp}$ and $\mathrm{SU}(5)_{\perp}$ spectral cover respectively. Moreover, we have examined all possible ways that the abelian factors undergoing monodromies are embedded in the enhanced symmetries at the points of double and triple intersections of seven branes. We have explored the implications of the successive U(1)-flux breaking of the $\mathrm{SO}(10)$ and $\mathrm{SU}(5)$ gauge symmetries down to the Standard Model gauge group. Using the combined data of the $\mathrm{U}(1)_{X}$ flux breaking $\mathrm{SO}(10) \rightarrow \mathrm{SU}(5) \times \mathrm{U}(1)_{X}$ and the $\mathrm{U}(1)_{Y}$ hypercharge flux breaking of SU(5) GUT down to SM, we have determined the induced restrictions on the multiplicities of the massless spectrum of the effective field theory models. Following the described procedure, we have built several examples of models and discussed their viability as well as their low energy massless spectrum. Despite the combined constraints arising form the GUT symmetries, monodromies and fluxes, we have seen that not all dangerous baryon couplings are eliminated, unless a matter parity is associated to the various massless states. We have considered the possibility that a discrete matter parity emanating from the geometric properties of the internal manifold can be communicated to the matter curves. We have confirmed the successful implementation of this geometric concept of matter parity in several examples constructed within the proposed scenario of this paper. Further investigations on the specific properties of these models would be required to discriminate them with respect to their low energy implications, however these are beyond the scope of the present work. We plan to revisit these interesting issues in a future publication.

\section{Acknowledgments}

This work was supported in part by the European Commission under the ERC Advanced Grant 226371 and the contract PITN-GA-2009-237920. The research project is co-financed by the European Union-European Social Fund (ESF) \& National Sources, in the framework of the program "THALIS" of the "Operational Program Education and Lifelong Learning" of the National Strategic Reference Framework (NSRF) 2007-2013. GKL would like to thank CERN, Theory Division for kind hospitality where part of this work has been carried out. 


\section{A F-SO(10) gauge symmetry and enhancements}

In this appendix we summarize some useful formulae and present the basic techniques for model building through the spectral cover approach. Furthermore, we examine in some detail the monodromic U(1)'s accompanying the $\mathrm{SO}(10)$ and $\mathrm{SU}(5)$ gauge symmetries.

We have seen that the $\mathrm{SO}(10)$ model has a spectral cover characterized by a $\mathrm{SU}(4)_{\perp}$ symmetry. We assume a single point of $\mathcal{E}_{8}$ enhanced symmetry with chiral matter and Higgs descending from the $\mathcal{E}_{8}$ - adjoint representation. Therefore, we start with the decomposition of the adjoint (2.2) under the breaking pattern (2.1). To obtain the effective $\mathrm{SO}(10)$ model, we further assume the breaking of $\mathrm{SU}(4) \rightarrow \mathrm{U}(1)^{3}$ by flux effects. Each of the $\mathrm{SO}(10)$ representations lies on a matter curve which is distinguished by the specific charge it carries under the $\mathrm{U}(1)^{3}$ Cartan subalgebra, characterized by the $\mathrm{SU}(4)_{\perp}$ weights denoted with $t_{i}, i=1,2,3,4$. As it happens for any $\mathrm{SU}(N)$ symmetry they satisfy the tracelessness condition

$$
t_{1}+t_{2}+t_{3}+t_{4}=0
$$

There are in principle four matter curves (denoted with $\Sigma_{16}$ ) accommodating the $\mathbf{1 6}$ representations and an equal number of $\overline{\mathbf{1 6}}$ matter curves, six $\Sigma_{10}$ matter curves for the $\mathbf{1 0}$, and fifteen singlets $\Sigma_{1}$. The $\mathrm{SU}(4)_{\perp}$ weights distinguishing the 16's, 10's and twelve singlets are given in (2.3). Then, the matter fields are localized on the curves which lie in the following directions of the Cartan subalgebra

$$
\text { 16: } t_{i}=0, \quad 10: t_{i}+t_{j}=0, \quad 1: t_{i}-t_{j}=0
$$

In $\mathrm{SO}(10)$ the Yukawa coupling giving mass to fermion fields is $\mathbf{1 6} \mathbf{1 6} \mathbf{1 0}$. Since the $\mathrm{SO}(10)$ representations carry $\mathrm{U}(1)_{i}$ charges, this coupling should also be invariant under these abelian factors

$$
16_{t_{i}} \mathbf{1 6}_{t_{j}} 10_{t_{k}+t_{l}}
$$

$\mathrm{U}(1)$ invariance is ensured by the condition $t_{i}+t_{j}+t_{k}+t_{l}=0$. Since these indices span the numbers $1,2,3,4$, this automatically implies that all indices $i, j, k, l$ differ from each other, so that we get

$$
t_{i}+t_{j}+t_{k}+t_{l}=t_{1}+t_{2}+t_{3}+t_{4}=0
$$

Thus, invariance under $\mathrm{U}(1)_{i} \in \mathrm{SU}(4)_{\perp}$ would require the 16's to descend from different matter curves. For example, if Higgs doublets are found in $\mathbf{1 0}_{t_{3}+t_{4}}$, then the only available couplings arise from $1 \mathbf{1 6}_{t_{1}} \mathbf{1 6}_{t_{2}} \mathbf{1 0}_{t_{3}+t_{4}}$, leading to off-diagonal tree level masses involving at least two generations. The known hierarchical fermion mass spectrum and the heaviness of the third generation however, is compatible with rank one structure of the mass matrices at tree-level. This requires a solution where at least two of the curves are identified through some (discrete) symmetry which has to be a subgroup of the Weyl group $W(\mathrm{SU}(4))=S_{4}$. For example, assuming the simplest case, namely a $\mathcal{Z}_{2}$ symmetry among $t_{1} \leftrightarrow t_{2}$, we obtain the identification $\mathbf{1 6} t_{t_{1}}=\mathbf{1 6} \boldsymbol{6}_{t_{2}}$. In this case we interpret $\mathbf{1 6} \mathbf{t}_{t_{1}} \mathbf{1 6}_{t_{2}} \mathbf{1 0}_{t_{3}+t_{4}}$ as a diagonal Yukawa coupling which provides masses to the third generation fermion fields. 


\begin{tabular}{|c|c|c|c|c|}
\hline $\operatorname{ord}(f)$ & $\operatorname{ord}(g)$ & $\operatorname{ord}(\Delta)$ & Fiber type & Singularity \\
\hline 0 & 0 & $n$ & $I_{n}$ & $A_{n-1}$ \\
$\geq 1$ & 1 & 2 & $I I$ & none \\
1 & $\geq 2$ & 3 & $I I I$ & $A_{1}$ \\
$\geq 2$ & 2 & 4 & $I V$ & $A_{2}$ \\
2 & $\geq 3$ & $n+6$ & $I_{n}^{*}$ & $D_{n+4}$ \\
$\geq 2$ & 3 & $n+6$ & $I_{n}^{*}$ & $D_{n+4}$ \\
$\geq 3$ & 4 & 8 & $I V^{*}$ & $E_{6}$ \\
3 & $\geq 5$ & 9 & $I I I^{*}$ & $E_{7}$ \\
$\geq 4$ & 5 & 10 & $I I^{*}$ & $E_{8}$ \\
\hline
\end{tabular}

Table 19. Kodaira's classification of Elliptic Singularities. The first three columns refer to the order of vanishing of $f, g, \Delta$ polynomials with respect to $z$. Column four denotes the type of the fiber ( $I$ nodal, $I I$ cuspidal etc.). Column five designates the associated singularity.

\section{A.1 Review of the Weierstrass form and some related material}

According to the 'standard' interpretation in F-theory the gauge symmetry is associated to the singularities of the internal compact manifold. A systematic analysis of these singularities has started with the work of Kodaira. Given the form of the Weierstrass equation

$$
y^{2}=x^{3}+f(z) x+g(z)
$$

the Kodaira classification relies on the vanishing order of the polynomials $f, g$ and the discriminant $\Delta$. This is summarized in table 19. A useful tool for the analysis of the gauge properties of an F-theory GUT is Tate's algorithm. Tate's Algorithm [42] provides a method to describe the singularities of the elliptic fiber and determine the local properties of the associated gauge group.

To study the semi-local model, we need to determine the properties of the matter curves and in particular how the elliptic fibration degenerates on the GUT surface $S_{\mathrm{GUT}}$. For local analysis, a suitable form of Weierstrass equation (Tate's form) is

$$
y^{2}+a_{1} x y+a_{3} y=x^{3}+a_{2} x^{2}+a_{4} x+a_{6}
$$

with $a_{n}$ being polynomial functions on the base. The indices of the coefficients $a_{n}$ have been chosen so to indicate the section they belong to, i.e. $a_{n} \in K_{B_{3}}^{-n}$. Thus each term is a section $K_{B_{3}}^{-6}$ (see [2] for details).

The standard form of the Weierstrass equation is

$$
y^{2}=x^{3}+f x+g
$$

and is obtained by completing the square and the cube, as follows. The square on the left hand side becomes

$$
\left(y+\frac{a_{1} x+a_{3}}{2}\right)^{2}=x^{3}+a_{2} x^{2}+a_{4} x+a_{6}+\left(\frac{a_{1} x+a_{3}}{2}\right)^{2}
$$


while we equate the r.h.s. with

$$
(x+\lambda)^{3}+f(x+\lambda)+g
$$

Comparing, we get

$$
\begin{aligned}
& f=\frac{1}{48}\left(24 a_{1} a_{3}-\left(a_{1}^{2}+4 a_{2}\right)^{2}\right)+a_{4} \\
& \begin{aligned}
g= & \frac{1}{864}\left(a_{1}^{6}+12 a_{1}^{4} a_{2}-36 a_{1}^{3} a_{3}+48 a_{1}^{2} a_{2}^{2}\right. \\
& \left.\quad-72 a_{4}\left(a_{1}^{2}+4 a_{2}\right)-144 a_{1} a_{2} a_{3}+64 a_{2}^{3}+216 a_{3}^{2}\right)+a_{6}
\end{aligned}
\end{aligned}
$$

Using the definitions

$$
\beta_{2}=a_{1}^{2}+4 a_{2}, \quad \beta_{4}=a_{1} a_{3}+2 a_{4}, \quad \beta_{6}=a_{3}^{2}+4 a_{6}
$$

the functions $f, g$ can be rewritten in a simpler form

$$
\begin{aligned}
f & =-\frac{1}{48}\left(\beta_{2}^{2}-24 \beta_{4}\right) \\
g & =-\frac{1}{864}\left(-\beta_{2}^{3}+36 \beta_{2} \beta_{4}-216 \beta_{6}\right)
\end{aligned}
$$

If we further define

$$
\beta_{8}=\beta_{2} a_{6}-a_{1} a_{3} a_{4}+a_{2} a_{3}^{2}-a_{4}^{2}
$$

we can write the discriminant

$$
\begin{aligned}
\Delta & =4 f^{3}+24 g^{2} \\
& =-\beta_{2}^{2} \beta_{8}-8 \beta_{4}^{3}-27 \beta_{6}^{2}+9 \beta_{2} \beta_{4} \beta_{6}
\end{aligned}
$$

$f, g$ are assumed to be functions of a complex coordinate $z$ on the base $B_{3}$.

We can now associate the vanishing order of $f(z), g(z), \Delta(z)$ to the singularity type of the compact manifold as in table 19. On the other hand, table 20 associates the coefficients $a_{n}$ to the singularity.

\section{A.2 $\mathrm{SO}(10)$}

We now apply the above analysis to the case of interest, namely the $\mathrm{SO}(10)$ model. Using the Tate's algorithm [42], for the $\mathrm{SO}(10)$ we substitute the coefficients $b_{i}$ with

$$
a_{1}=-b_{5} z, \quad a_{2}=b_{4} z, \quad a_{3}=-b_{3} z^{2}, \quad a_{4}=b_{2} z^{3}, \quad a_{6}=b_{0} z^{5}
$$

and we can write the Weierstrass equation as follows

$$
y^{2}=x^{3}+b_{5} x y z+b_{4} x^{2} z+b_{3} y z^{2}+b_{2} x z^{3}+b_{0} z^{5}
$$

We mention that $x, y$ are homogeneous coordinates of the torus fiber and $b_{i}$ functions of the coordinates of the three-fold base. The coefficients $b_{i}$ are non-vanishing and may have subleading terms being powers of $z$. We can study the discriminant and determine the singularity enhancements along the lines of ref [2]. 


\begin{tabular}{|c|c|c|c|c|c|c|c|}
\hline Type & Group & $a_{1}$ & $a_{2}$ & $a_{3}$ & $a_{4}$ & $a_{6}$ & $\Delta$ \\
\hline$I_{0}$ & 0 & 0 & 0 & 0 & 0 & 0 & 0 \\
\hline$I_{1}$ & - & 0 & 0 & 1 & 1 & 1 & 1 \\
\hline$I_{2}$ & - & 0 & 0 & 1 & 1 & 2 & 2 \\
\hline$I_{2 n}^{s}$ & $\mathrm{SU}(2 n)$ & 0 & 1 & $n$ & $n$ & $2 n$ & $2 n$ \\
\hline$I_{2 n+1}^{s}$ & $\mathrm{SU}(2 n+1)$ & 0 & 1 & $n$ & $n+1$ & $2 n+1$ & $2 n+1$ \\
\hline$I_{1}^{* s}$ & $\mathrm{SO}(10)$ & 1 & 1 & 2 & 3 & 5 & 7 \\
\hline$I_{2 k-3}^{* s}$ & $\mathrm{SO}(4 k+2)$ & 1 & 1 & $k$ & $k+1$ & $2 k+1$ & $2 k+3$ \\
\hline$I V^{* s}$ & $E_{6}$ & 1 & 2 & 3 & 3 & 5 & 8 \\
\hline$I I I^{* S}$ & $E_{7}$ & 1 & 2 & 3 & 3 & 5 & 9 \\
\hline$I I^{s}$ & $E_{8}$ & 1 & 2 & 3 & 4 & 5 & 10 \\
\hline
\end{tabular}

Table 20. Partial results of Tate's Algorithm. (The complete results can be found in [56].) The order of vanishing of the coefficients $a_{i} \sim z^{n_{i}}$ and the corresponding gauge group. The highest singularity allowed in the elliptic fibration is $E_{8}$.

For the local picture (i.e. in the limit $z=0$ ) we recall that the $b_{i}$-subleading terms vanish and $b_{i}$ become constants that can be interpreted as sections of line bundles on the surface $S_{\mathrm{GUT}}$. The discriminant is

$$
\begin{aligned}
\Delta= & -\left(16 b_{3}^{2} b_{4}^{3}\right) z^{7} \\
& +\left(27 b_{3}^{4}-36 b_{4} b_{5} b_{3}^{3}+8 b_{4}\left(b_{4} b_{5}^{2}-9 b_{2}\right) b_{3}^{2}-16 b_{2} b_{4}^{2} b_{5} b_{3}+16 b_{4}^{2}\left(4 b_{0} b_{4}-b_{2}^{2}\right)\right) z^{8} \\
+ & {\left[64 b_{2}^{3}-8 b_{5}\left(b_{4} b_{5}-12 b_{3}\right) b_{2}^{2}-2\left(b_{3}\left(4 b_{4} b_{5}-15 b_{3}\right) b_{5}^{2}+144 b_{0} b_{4}\right) b_{2}\right.} \\
& \left.+b_{3}^{2} b_{5}^{3}\left(b_{4} b_{5}-b_{3}\right)+24 b_{0}\left(9 b_{3}^{2}-6 b_{4} b_{5} b_{3}+2 b_{4}^{2} b_{5}^{2}\right)\right] z^{9}+\cdots
\end{aligned}
$$

while the functions $f, g$ are

$$
\begin{aligned}
& f(z)=\frac{1}{48}\left(24\left(2 b_{2}+b_{3} b_{5}\right) z^{3}-\left(z^{2} b_{5}^{2}+4 z b_{4}\right)^{2}\right) \\
& g(z)=\frac{1}{864}\left(\left(z^{2} b_{5}^{2}+4 z b_{4}\right)^{3}-36\left(2 b_{2} z^{3}+b_{3} b_{5} z^{3}\right)\left(z^{2} b_{5}^{2}+4 z b_{4}\right)+216\left(4 b_{0} z^{5}+b_{3}^{2} z^{4}\right)\right)
\end{aligned}
$$

\section{A.2.1 Symmetry enhancement}

From the above formulae we see that the coefficients in the lowest powers of $z$ are

$$
\begin{aligned}
& f(z)=-\frac{b_{4}^{2}}{3} z^{2}+\frac{1}{48}\left(-8 b_{4} b_{5}^{2}+24 b_{3} b_{5}+48 b_{2}\right) z^{3}+\mathcal{O}\left(z^{4}\right), \\
& g(z)=\frac{2 b_{4}^{3}}{27} z^{3}+\mathcal{O}\left(z^{4}\right)
\end{aligned}
$$

Clearly, we can see from table 19 that this is indeed a $D_{5}=\mathrm{SO}(10)$ singularity since they satisfy $\operatorname{deg} f=2, \operatorname{deg} g=3$ and $\operatorname{deg} \Delta=7$. There are several ways to enhance this symmetry:

i) Setting $b_{4}=0$, to lowest order in $z$ we get

$$
f(z)=\left(b_{2}+\frac{b_{3} b_{5}}{2}\right) z^{3}+\mathcal{O}\left(z^{4}\right), \quad g(z)=\frac{b_{3}^{2}}{4} z^{4}+\mathcal{O}\left(z^{5}\right), \quad \Delta(z)=b_{3}^{4} z^{8}+\mathcal{O}\left(z^{9}\right)
$$


According to Kodaira's classification along this intersection we get an $\mathcal{E}_{6}$ enhancement of the $\mathrm{SO}(10)$ singularity where the $\mathbf{1 6}$ of $\mathrm{SO}(10)$ resides. This can be seen from the decomposition

$$
27 \rightarrow \mathbf{1 6}_{1}+\mathbf{1 0}_{-2}+\mathbf{1}_{4}
$$

Next, we consider a second order enhancement with the case when both $b_{3}=b_{4}=0$. We obtain

$f(z)=b_{2} z^{3}+\mathcal{O}\left(z^{4}\right), \quad g(z)=\left(b_{0}-\frac{1}{12} b_{2} b_{5}^{2}\right) z^{5}+\mathcal{O}\left(z^{6}\right), \quad \Delta(z)=-64 b_{2}^{3} z^{9}+\mathcal{O}\left(z^{10}\right)$

Thus, this corresponds to an $E_{7}$ enhancement. Decomposition of the $E_{7}$ representations

$$
56 \rightarrow 27+\overline{27}+1+1, \quad 133 \rightarrow 78+27+\overline{27}+1
$$

entails the realization of the $\mathrm{SO}(10)$ Yukawa coupling 161610 , since

$$
56 \cdot 56 \cdot 133 \rightarrow 27 \cdot 27 \cdot(27+78) \rightarrow 16_{1} \cdot 16_{1} \cdot 10_{-2}
$$

ii) We may study other types of enhancements as follows. Consider first that $b_{3}=0$. Then, to lowest order in $z$, we have

$f(z)=-\frac{b_{4}^{2}}{3} z^{2}+\mathcal{O}\left(z^{3}\right), \quad g(z)=\frac{2 b_{4}^{3}}{27} z^{3}+\mathcal{O}\left(z^{4}\right), \quad \Delta(z)=16 b_{4}^{2}\left(b_{2}^{2}-4 b_{0} b_{4}\right) z^{8}+\mathcal{O}\left(z^{9}\right)$

which corresponds to the enhancement $D_{6}=\mathrm{SO}(12)$. We have the following decompositions along this singularity enhancement

$$
66 \rightarrow 45+1+10_{2}+\overline{10}_{2}, \quad 32 \rightarrow 16_{1}+\overline{16}_{-1}, \quad 12 \rightarrow 10_{0}+1_{2}+1_{-2}
$$

Thus Higgs fields are found in the decomposition of 66 adjoint while half of 32 representation corresponds to $\mathbf{1 6}$.

This singularity is further enhanced to $D_{7}=\mathrm{SO}(14)$ if in addition to $b_{3}=0$ we also impose

$$
b_{2}^{2}-4 b_{0} b_{4}=0 \rightarrow \operatorname{deg} \Delta=9
$$

The SO(12) representations are found in the decompositions

$$
14 \rightarrow 12+1+1, \quad 91 \rightarrow 66+1+12+12^{\prime}
$$

Now, let us examine how this singularity looks like locally. A way to obtain a local model from a global one, is to assign scaling dimensions to $(x, y, z)$ and retain only the relevant terms. To this end, we introduce the scaling dimensions $[2,28](x, y, z) \sim\left(\frac{1}{3}, \frac{1}{2}, \frac{1}{5}\right)$ and observe that the terms of order one recreate the $E_{8}$ singularity

$$
y^{2}=x^{3}+b_{0} z^{5}
$$


The term $b_{5} x y z$ is of order higher than one while all the remaining all less than one. Dropping the term with scaling greater than one we obtain the local deformation of the $E_{8}$ singularity

$$
y^{2}=x^{3}+b_{4} x^{2} z+b_{3} y z^{2}+b_{2} x z^{3}+b_{0} z^{5}
$$

which does not depend on $b_{5}$.

We can see how this is 'encoded' locally into an SU(4) spectral cover along the intersection curve by identifying $z^{5}$ with the fourth power of an affine parameter $s$

$$
z^{5} \equiv s^{4} \rightarrow z=s^{4 / 5}
$$

Let now fix the $x$ scaling with respect to $s$ by demanding that the term of $b_{2}$ coefficient is a power of $s^{2}$

$$
x z^{3}=s^{2} \rightarrow x=s^{2} z^{-3}=s^{-2 / 5}
$$

We further require that the $b_{3}$ coefficient multiplies the first power of $s$, thus

$$
y z^{2}=s \rightarrow y=s z^{-2}=s^{-3 / 5}
$$

All remaining terms are now fixed. In particular we find also that $y^{2}=s^{-6 / 5}=x^{3}$. In the spectral cover equation (A.9) now becomes

$$
0=b_{4}+b_{3} s+b_{2} s^{2}+b_{0} s^{4}
$$

This is indeed an $\mathrm{SU}(4)$ spectral cover with the $b_{i}$ coefficients as in (A.9). ( $b_{1}=0$ as expected for any $\mathrm{SU}(N)$.)

\section{A.2.2 Extraneous matter}

In dealing with $\mathrm{SO}(10)$ models one has to take into account the extraneous matter which eventually appears in the bulk. In order to study a sufficiently general case, we consider a seven-brane with worldvolume gauge group $E_{6}$. The bulk matter content all descends from the adjoint representation 78 which in the case of $\mathrm{SO}(10)$ decomposes to the adjoint representation $\mathbf{4 5}$ as well as the $\mathbf{1 6}$ and $\overline{\mathbf{1 6}}$. Under the successive $E_{6} \rightarrow \mathrm{SO}(10) \times \mathrm{SU}(3)$ and $\mathrm{SO}(10) \rightarrow \mathrm{SU}(5) \times \mathrm{U}(1)_{X}$ breaking we get

$$
\begin{aligned}
(\mathbf{7 8}, 1) \rightarrow & (\mathbf{4 5}, 1)_{0}+(\mathbf{1}, 1)_{0}+(\mathbf{1 6}, 1)_{-3}+(\overline{\mathbf{1 6}}, 1)_{3} \\
\rightarrow & {\left[(24,1)_{0,0}+(1,1)_{0,0}+10_{4,0}+\overline{10}_{-4,0}\right]+(1,1)_{0} } \\
& +\left[10_{(-1,-3)}+\overline{5}_{(3,-3)}+1_{(-5,-3)}\right]+\left[\overline{10}_{(1,3)}+5_{(-3,3)}+1_{(5,3)}\right]
\end{aligned}
$$

This decomposition determines the matter on the bulk surface $S$.

Define the line bundles

$$
\mathcal{L}_{1}=(4,0), \quad \mathcal{L}_{2}=(-1,-3),
$$

Then the multiplicity of the various representations emerging from 78 are computed by a topological index formula (Euler characteristic $\chi$ ) as follows. Let $a_{1}, b_{1}$ the multiplicities 
of $10_{4,0}, \overline{10}_{-4,0}$ and $a_{2}, b_{2}$ those of $10_{(-1,-3)}, \overline{10}_{(1,3)}$ respectively. These are given by

$$
\begin{aligned}
a_{j} & =-\chi\left(\mathcal{L}_{j}, S\right)=1+\frac{1}{2} c_{1}\left(\mathcal{L}_{j}\right) \cdot\left(c_{1}\left(\mathcal{L}_{j}\right)+c_{1}(S)\right) \\
b_{j} & =-\chi\left(\mathcal{L}_{j}^{-1}, S\right)=1+\frac{1}{2} c_{1}\left(\mathcal{L}_{j}^{-1}\right) \cdot\left(c_{1}\left(\mathcal{L}_{j}^{-1}\right)+c_{1}(S)\right)
\end{aligned}
$$

which imply

$$
a_{j}+b_{j}=2+c_{1}\left(\mathcal{L}_{j}\right)^{2}, \quad a_{j}-b_{j}=c_{1}(S) \cdot c_{1}\left(\mathcal{L}_{j}\right)
$$

We observe that vector like multiplets arise if $a_{j}-b_{j}=0$ which implies the restriction

$$
c_{1}(S) \cdot c_{1}\left(\mathcal{L}_{j}\right)=0
$$

Furthermore, the remaining can be expressed as linear combinations

$$
\begin{aligned}
& \kappa=\# \text { of } \overline{5}_{(3,-3)}=-\chi\left(\mathcal{L}_{1} \otimes \mathcal{L}_{2}\right) \\
& \lambda=\# \text { of } 5_{(-3,3)}=-\chi\left(\mathcal{L}_{1}^{-1} \otimes \mathcal{L}_{2}^{-1}\right) \\
& \mu=\# \text { of } 1_{(-5,-3)}=-\chi\left(\mathcal{L}_{1}^{-1} \otimes \mathcal{L}_{2}\right) \\
& \nu=\# \text { of } 1_{(5,3)}=-\chi\left(\mathcal{L}_{1} \otimes \mathcal{L}_{2}^{-1}\right)
\end{aligned}
$$

leading to

$$
\begin{aligned}
& \kappa+\lambda=2+c_{1}\left(\mathcal{L}_{1}\right)^{2}+c_{1}\left(\mathcal{L}_{2}\right)^{2} \\
& \kappa-\lambda=c_{1}(S) \cdot c_{1}\left(\mathcal{L}_{1}\right)+c_{1}(S) \cdot c_{1}\left(\mathcal{L}_{2}\right) \\
& \mu+\nu=2+c_{1}\left(\mathcal{L}_{1}\right)^{2}+c_{1}\left(\mathcal{L}_{2}\right)^{2} \\
& \mu-\nu=-c_{1}(S) \cdot c_{1}\left(\mathcal{L}_{1}\right)+c_{1}(S) \cdot c_{1}\left(\mathcal{L}_{2}\right)
\end{aligned}
$$

Clearly, we can satisfy the criteria for vector-like pairs discussed above, however there is no choice of $c_{1}$ 's to eliminate all of these states.

\section{A.3 Polynomial equations for the matter curves}

We can proceed with the analysis of the SO(10) GUT models using the equivalent description of the spectral cover approach. More precisely we can describe the model in the context of the Higgs bundle picture which is given in terms of the adjoint scalars and the gauge field.

We have seen that for the $E_{8}$ embedding of the $\mathrm{SO}(10)$ singularity the commutant is $\mathrm{SU}(4)$, while this is given by the hypersurface

$$
\mathcal{C}_{4}=\sum_{k=1}^{4} b_{k} s^{4-k}=b_{0} s^{4}+b_{1} s^{3}+b_{2} s^{2}+b_{3} s+b_{4}=0
$$

with $s$ being an affine parameter and $b_{1}=0$. This is the spectral cover for the fundamental representation of SU(4). We denote with $c_{1}$ the $1^{\text {st }}$ Chern class of the Tangent Bundle to $S_{\mathrm{GUT}}$ and $-t$ the $1^{\text {st }}$ Chern class of the Normal Bundle to $S_{\mathrm{GUT}}$. It is customary to define the following quantity

$$
\eta=6 c_{1}-t
$$


Using this, we can express the coefficients $b_{k}, k=0, \ldots, 4$ as sections of

$$
\left[b_{k}\right]=\eta-k c_{1}=(6-k) c_{1}-t
$$

while $[s]=-c_{1}$, so that each term in (A.12) is $\left[b_{k} s^{4-k}\right]=\eta-4 c_{1}$.

We can determine the 'locations' $t_{i}$ of the four $\mathbf{1 6}$ representations as the roots of the polynomial

$$
\begin{aligned}
P_{16}(s) & =b_{0}\left(s-t_{1}\right)\left(s-t_{2}\right)\left(s-t_{3}\right)\left(s-t_{4}\right) \\
& =b_{0} s^{4}+b_{1} s^{3}+b_{2} s^{2}+b_{3} s+b_{4}
\end{aligned}
$$

We can identify the parameter $s$ with the Higgs vev breaking $\mathcal{E}_{8}$. Setting $s=0$, we can see that the equation

$$
P_{16}(s=0)=0 \Rightarrow b_{4}=t_{1} t_{2} t_{3} t_{4}=0
$$

determines the 'locations' of the four matter curves which lift to a single one in the spectral cover.

It is useful to derive the equations relating coefficients $b_{k}$ and $t_{i}$. These are

$$
\begin{aligned}
& b_{1}=-b_{0}\left(t_{1}+t_{2}+t_{3}+t_{4}\right)=0 \\
& b_{2}=b_{0}\left(t_{1}^{2}+t_{2}^{2}+t_{3}^{2}+t_{1} t_{2}+t_{2} t_{3}+t_{3} t_{1}\right) \\
& b_{3}=b_{0}\left(t_{1}+t_{2}\right)\left(t_{1}+t_{3}\right)\left(t_{2}+t_{3}\right) \\
& b_{4}=b_{0} t_{1} t_{2} t_{3} t_{4}=-b_{0} t_{1} t_{2} t_{3}\left(t_{1}+t_{2}+t_{3}\right)
\end{aligned}
$$

where the solution of $b_{1}=0 \rightarrow t_{4}=-\left(t_{1}+t_{2}+t_{3}\right)$ has been substituted into $b_{2,3,4}$.

Next we construct the spectral cover for the antisymmetric representation $\mathbf{1 0} \in \mathrm{SO}(10)$. The 10 representations are characterized by the weights $t_{i}+t_{j}$ with $i, j=1,2,3,4$. Proceeding as in the case of the 16's, we can write the equation for the 10's of $\mathrm{SO}(10)$ as follows:

$$
\begin{aligned}
P_{10}(s) & =b_{0}^{2} \prod_{i<j}\left(s+t_{i}+t_{j}\right) \\
& =b_{0}^{2}\left(s-t_{1}-t_{2}\right)\left(s+t_{1}+t_{2}\right)\left(s-t_{1}-t_{3}\right)\left(s+t_{1}+t_{3}\right)\left(s-t_{2}-t_{3}\right)\left(s+t_{2}+t_{3}\right) \\
& =b_{0}^{2} s^{6}+c_{1} s^{5}+c_{2} s^{4}+s_{3} s^{3}+c_{4} s^{2}+c_{5} s+c_{6}
\end{aligned}
$$

For later use, we express $c_{n}$ in terms of $t_{i}$ and then using equations (A.15) we convert them to functions of $b_{k}$

$$
\begin{aligned}
& c_{2}=-2 b_{0}^{2}\left(t_{1}^{2}+t_{2}^{2}+t_{3}^{2}+t_{1} t_{2}+t_{2} t_{3}+t_{3} t_{1}\right) b_{0}^{2}=-2 b_{2} b_{0}^{2} \\
& c_{6}=-b_{0}^{2}\left(t_{1}+t_{2}\right)^{2}\left(t_{1}+t_{3}\right)^{2}\left(t_{2}+t_{3}\right)^{2}=-b_{3}^{2} \\
& c_{4}=b_{0}^{2}\left(t_{1}^{4}+2\left(t_{2}+t_{3}\right) t_{1}^{3}+\left(3 t_{2}^{2}+8 t_{3} t_{2}+3 t_{3}^{2}\right) t_{1}^{2}\right. \\
& \left.\quad \quad+2\left(t_{2}+t_{3}\right)\left(t_{2}^{2}+3 t_{3} t_{2}+t_{3}^{2}\right) t_{1}+\left(t_{2}^{2}+t_{3} t_{2}+t_{3}^{2}\right)^{2}\right)
\end{aligned}
$$

and the last one can be rewritten

$$
c_{4}=b_{2}^{2}+4 b_{0}^{2} t_{1} t_{2} t_{3}\left(t_{1}+t_{2}+t_{3}\right) \equiv b_{2}^{2}-4 b_{4} b_{0}
$$




\begin{tabular}{|c|l|c|}
\hline Order & \multicolumn{1}{|c|}{ Equation } & Enhancement \\
\hline $1^{\text {st }}$ & $b_{4}=0$ & $E_{6}$ \\
$2^{\text {nd }}$ & $b_{4}=b_{3}=0$ & $E_{7}$ \\
\hline $1^{\text {st }}$ & $b_{3}=0$ & $\mathrm{SO}(12)$ \\
$2^{\text {nd }}$ & $b_{3}=b_{2}^{2}-4 b_{0} b_{4}=0$ & $\mathrm{SO}(14)$ \\
\hline
\end{tabular}

Table 21. First and second order enhancements of $\mathrm{SO}(10)$. The $b_{i}$ are the coefficients of the corresponding Weierstrass equation (A.5).

Thus, all coefficients are in terms of $b_{i}$ 's and $P_{10}$ takes the simple form

$$
P_{10}(s)=b_{0}^{2} s^{6}-2 b_{2} b_{0} s^{4}+\left(b_{2}^{2}-4 b_{4} b_{0}\right) s^{2}-b_{3}^{2}
$$

Setting $s=0$, we see then that the 7-branes associated to 10's of SO(10) are determined by

$$
b_{3}^{2}=0
$$

The corresponding first and second order enhancements of $\mathrm{SO}(10)$ are collected in table 21.

\section{B Monodromies}

Much of the F-theory edifice rests on the notion of monodromies. Matter curves are associated to the roots $t_{i}$ which are polynomial solutions with factors combinations of $b_{i}$ 's, thus

$$
b_{i}=b_{i}\left(t_{j}\right)
$$

Generically, the inversion of these equations will lead to branchcuts and he solutions $t_{j}=t_{j}\left(b_{i}\right)$ are subject to monodromy actions. There are several ways to factorize the spectral cover equation, the most obvious possibilities are $2+1+1,2+2$ and $3+1$ corresponding to $(2.6),(2.17)$ and $(2.23)$ respectively. For the first case we have a single $\mathcal{Z}_{2}$ monodromy among $\left\{t_{1}, t_{2}\right\}$. In the second case we get the identifications between $\left\{t_{1}, t_{2}\right\}$ and similarly among $\left\{t_{3}, t_{4}\right\}$ implying a $\mathcal{Z}_{2} \times \mathcal{Z}_{2}$ monodromy and finally in case 3 we have a $\mathcal{Z}_{3}$ monodromy among $\left\{t_{1}, t_{2}, t_{3}\right\}$. Next we analyze in detail these three cases.

\section{B.1 Symmetry enhancements and $\mathcal{Z}_{2}$ monodromy}

The spectral cover equation for the factorization $C_{2+1+1}$ is given in (2.6) and corresponds to a $\mathcal{Z}_{2}$ monodromy among $t_{1} \leftrightarrow t_{2}$. Putting $s=0$ we find

$$
P_{16}(0)=a_{1} a_{4} a_{6}=0
$$

thus, there are three $\mathbf{1 6}$ 's left after the monodromy action which are in

$$
a_{1}=0, \quad a_{4}=0, \quad a_{6}=0
$$




\begin{tabular}{|c|c|c|c|c|c|c|}
\hline$a_{1}$ & $a_{2}$ & $a_{3}$ & $a_{4}$ & $a_{5}$ & $a_{6}$ & $a_{7}$ \\
$\eta-2 c_{1}-\chi$ & $\eta-c_{1}-\chi$ & $\eta-\chi$ & $-c_{1}+x_{5}$ & $x_{5}$ & $-c_{1}+x_{7}$ & $x_{7}$ \\
\hline
\end{tabular}

Table 22. Homology classes for coefficients $a_{i}$ for the $\mathcal{Z}_{2}$ case.

The determination of the $a_{i}$ homologies can be achieved through their link to the known $b_{k}$ 's. Comparing powers of $s$ between (A.12) and (2.6) we get

$$
\begin{aligned}
& b_{4}=a_{1} a_{4} a_{6} \\
& b_{3}=a_{2} a_{4} a_{6}+a_{1} a_{5} a_{6}+a_{1} a_{4} a_{7} \\
& b_{2}=a_{3} a_{4} a_{6}+a_{2} a_{5} a_{6}+a_{2} a_{4} a_{7}+a_{1} a_{5} a_{7} \\
& b_{1}=a_{3} a_{5} a_{6}+a_{3} a_{4} a_{7}+a_{2} a_{5} a_{7} \\
& b_{0}=a_{3} a_{5} a_{7}
\end{aligned}
$$

We solve the constraint $b_{1}=0$ adopting the following Ansatz:

$$
a_{3}=\lambda a_{5} a_{7}, \quad a_{2}=-\lambda\left(a_{5} a_{6}+a_{4} a_{7}\right)
$$

Given that the coefficients $b_{k}$ satisfy (A.13), for the specific combinations of indices appearing in (B.1) we have

$$
\eta-k c_{1}=\left[a_{l}\right]+\left[a_{m}\right]+\left[a_{n}\right], \quad \text { with } \quad k+l+m+n=15
$$

where $l, m, n$ take the values $1,2, \ldots, 7$ and $k=0,1,2,3,4$. These are five equations with seven unknowns. We choose two arbitrary values $\left[a_{5}\right]=\chi_{5},\left[a_{7}\right]=\chi_{7}$ while for convenience we introduce

$$
\chi=\chi_{5}+\chi_{7}
$$

and solve the system. The results are presented in table 22. The homology of $\lambda$ can be specified using the homologies of $a_{i}$ presented in table 22 :

$$
[\lambda]=\eta-2 \chi=6 c_{1}-t-2 \chi
$$

Substituting the solution for $b_{1}=0$, the $b_{k}$ coefficients become

$$
\begin{aligned}
& b_{0}=\lambda\left(a_{5} a_{7}\right)^{2} \\
& b_{1}=0 \\
& b_{2}=a_{5} a_{7}\left(a_{1}+\lambda a_{4} a_{6}\right)-\lambda\left(a_{5} a_{6}+a_{4} a_{7}\right)^{2} \\
& b_{3}=\left(a_{1}-\lambda a_{4} a_{6}\right)\left(a_{5} a_{6}+a_{4} a_{7}\right) \\
& b_{4}=a_{1} a_{4} a_{6}
\end{aligned}
$$

We have already investigated the coefficients $c_{i}\left(b_{k}\right)$ of the polynomial associated to $\Sigma_{10}$ matter curves and found that their equations satisfy $b_{3}^{2}=0$. Therefore their homologies are specified by the homologies of the factors constituting $b_{3}$. Notice that the coefficient $b_{3}$ appearing in the above solution is already factorized, its factors written as simple combinations of $a_{i}$. Since all $\left[a_{i}\right]$ classes are specified, it is straightforward to see that equation 


\begin{tabular}{|c|c|c|c|}
\hline $\mathbf{1 0}$ & $\left(a_{1}-\lambda a_{4} a_{6}\right)$ & $\eta-2 c_{1}-\chi$ & $t_{1,2}+t_{3} / t_{4}$ \\
$\mathbf{1 0}$ & $\left(a_{5} a_{6}+a_{4} a_{7}\right)$ & $-c_{1}+\chi$ & $t_{3}+t_{4}$ \\
\hline
\end{tabular}

Table 23. Homology classes of $\mathbf{1 0}$ 's for the $\mathcal{Z}_{2}$ case.

$b_{3}^{2}=0$ defines four 10-matter curves with homology classes determined straightforwardly from those of $a_{i}$ 's and given in table 23. On the other hand we know that under the $t_{1} \leftrightarrow t_{2}$ identification the six 10's characterized by the $t_{i}+t_{j}$ reduce to four, in accordance with the factorization of $b_{3}$. We collect all the results in table 1 .

\section{B.1.1 Enhancements along intersecting matter curves}

We have seen previously how the various singularity enhancements along matter curves are attributed to the vanishing of some $b_{i}$ 's. Here we will associate these enhancements directly to the coefficients $a_{i}$. We note that this analysis can help us specify exactly which U(1)'s are embedded in the enhanced gauge group. Further, since it happens that the coefficients $a_{i}$ are directly related to monodromies, this might be useful in phenomenological applications. For example, the computation of the Yukawa couplings requires knowledge of the wavefunctions of the states participating at the triple intersection. The wavefunction profiles are determined by the solution of a set of differential equations obtained from varying the equations of motion [3]. The computation of the wavefunctions and the Yukawa coupling depend crucially on the whether $\mathrm{U}(1)$ in the intersection undergo monodromies or not $[26]$.

$\boldsymbol{E}_{6}$ and $\boldsymbol{E}_{7}$ enhancement. We know already that this enhancement is obtained setting $b_{4}=0$. At the level of the $a_{i}$ coefficients this can be done by demanding either of $a_{1,4,6}$ to be zero. There is a difference however between $a_{1}=0$ and $a_{4,6}=0$. The first case (i.e. $a_{1}=0$ which is involved in the two $t_{1,2}$ undergoing a monodromy) implies

$$
s a_{2}+s^{2} a_{3}=s\left(a_{2}+a_{3} s\right)
$$

This means that for the particular choice $a_{1}=0$ at the first order enhancement to $E_{6}$ the monodromy is 'resolved' in the sense that one of the two $\mathrm{U}(1)$ involved $(s=0)$ is incorporated to the $E_{6}$ symmetry.

Here, we make the general observation that if the monodromy is among U(1)'s (or the $t_{a}$ 's) which are embedded into the $E_{6}$ enhancement, at this enhanced symmetry level we have distinct $27_{t_{1,2,3}}$ matter curves and the corresponding wavefunctions refer to different entities at this stage. On the contrary, if the monodromy is in $\mathrm{U}(1)_{i} \in \mathrm{SU}(3)_{\perp}$ then some $27_{t_{i}}$ are identified. We reckon that these distinct cases might have some relevance on the determination of the wavefunctions of the states participating in the vertex and as a consequence to the Yukawa coupling computations [26], however we leave such an analysis for a future work.

For the other two cases $\left(a_{4,6}=0\right)$ the monodromy among the two U(1)'s is preserved at the first order enhancement of the symmetry. 
- The condition $b_{4}=0$ is satisfied by setting any of $a_{1}, a_{4}, a_{6}$ zero. Thus, let $a_{1}=0$. Checking the discriminant and the vanishing order of the coefficients in Weierstrass equation, we find

$$
\begin{aligned}
f(z) & =\left(\lambda a_{4} a_{5} a_{6} a_{7}-\lambda\left(a_{5} a_{6}+a_{4} a_{7}\right)^{2}\right) z^{3}+\cdots, \\
g(z) & =\frac{1}{4} \lambda^{2} a_{4}^{2} a_{6}^{2}\left(a_{5} a_{6}+a_{4} a_{7}\right)^{2} z^{4}+\cdots \\
\Delta(z) & =-27 \lambda^{4} a_{4}^{4} a_{6}^{4}\left(a_{5} a_{6}+a_{4} a_{7}\right)^{4} z^{8}+\cdots
\end{aligned}
$$

that is, we obtain an $E_{6}$ enhancement.

In addition we set now $b_{3}=0$, which, as we have seen enhances the symmetry to $E_{7}$. With respect to $a_{i}$, this happens when either of the following occurs:

$$
a_{4}=0, \quad a_{6}=0, \quad a_{5} a_{6}+a_{4} a_{7}=0
$$

a) The first two cases are equivalent. Accepting $a_{4}=0$ the spectral cover equation becomes

$$
\mathcal{C}_{4}=s^{2}\left(a_{2}+a_{3} s\right)\left(a_{6}+a_{7} s\right)
$$

Then, the functions $f, g$ and the discriminant $\Delta$ become

$$
f(z)=-\lambda a_{5} a_{6} z^{3}, \quad g(z) \sim z^{5}, \quad \Delta \sim z^{9}
$$

which upgrades the symmetry to $E_{7} \supset E_{6} \times \mathrm{U}(1)$ whose fundamental decomposes

$$
56 \rightarrow 27+\overline{27}+1+1
$$

b) The case $a_{5} a_{6}+a_{4} a_{7}=0$. This simultaneously implies $a_{2}=0$, as can be observed from the inspection of the Ansatz (B.2). Together with the previous condition $a_{1}=0$ implies

$$
\left(a_{4}+a_{5} s\right)\left(a_{6}+a_{7} s\right) a_{3} s^{2}=0
$$

If we solve $a_{5} a_{6}+a_{4} a_{7}=0$ assuming $a_{5}=\lambda a_{4}, a_{7}=-\lambda a_{6}$,

$$
a_{3} a_{4} a_{6}(1+\lambda s)(1-\lambda s) s^{2}=0
$$

which implies $\left(a_{3} s^{2}=0\right)$ that both U(1)'s involved in the monodromy descend from $E_{7}$ :

$$
E_{7} \supset E_{6} \times \mathrm{U}(1) \supset \mathrm{SO}(10) \times \mathrm{U}(1) \times \mathrm{U}(1)^{\prime}
$$

- The $E_{6}$ enhancement of course occurs also if $a_{4}=0$ (and similarly if $a_{6}=0$ ). Notice that the $\mathcal{Z}_{2}$ monodromy is among the $t_{1,2}$ which are associated to the coefficients $a_{1,2,3}$ and thus it is now unaffected. This means that the U(1)'s associated to $a_{4}$ (or $a_{6}$ ) coefficients are incorporated into the $E_{6}$ symmetry. Therefore the monodromy occurs among the U(1)'s emerging from the commutant of $E_{6}$ :

$$
E_{8} \rightarrow E_{6} \times \mathrm{SU}(3)
$$




\begin{tabular}{|c|c|c|}
\hline$E_{6}$ & $E_{7}$ & Monodromy \\
\hline$a_{1}=0$ & $a_{4} / a_{6}=0$ & $t_{1,2} \in E_{6} \times \mathrm{SU}(3)$ \\
$a_{1}=0$ & $a_{5} a_{6}+a_{4} a_{7}=0$ & $t_{1,2} \in E_{7}$ \\
$a_{4}=0$ & $a_{6}=0$ & $\tilde{t}_{1,2} \in \mathrm{SU}(3)$ \\
$a_{6}=0$ & $a_{4}=0$ & $\tilde{t}_{1,2} \in \mathrm{SU}(3)$ \\
\hline
\end{tabular}

Table 24. The vanishing coefficients with the corresponding enhancements and the embedding of the U(1)'s involved in the monodromy. In the first case the monodromy is between a $\mathrm{U}(1) \in E_{6}$ and $\mathrm{U}(1) \in \mathrm{SU}(3)$. In the last two cases, the monodromy is among the $\mathrm{U}(1)$ 's in the orthogonal complement of $E_{6}$ implying identification of the 27 's.

We proceed with the discriminant and $f, g$ functions which for $a_{4}=0$ are given by

$$
\begin{aligned}
f(z) & =\left(a_{1} a_{5} a_{7}-\lambda a_{5}^{2} a_{6}^{2}\right) z^{3}+\cdots, \\
g(z) & =\frac{1}{4} a_{1}^{2} a_{5}^{2} a_{6}^{2} z^{4}+\cdots \\
\Delta(z) & =-27 a_{1}^{4} a_{5}^{4} a_{6}^{4} z^{8}+\cdots
\end{aligned}
$$

a) Let now $a_{1}=0$. The case reduces to the previous one of $E_{7}$ enhancement an in (B.4).

b) Let $a_{6}=0$. Then

$$
f(z)=a_{1} a_{5} a_{7} z^{3}+\cdots, \quad g(z)=\lambda a_{5}^{2} a_{7}^{2} z^{5}+\cdots, \quad \Delta(z)=-64 a_{1}^{3} a_{5}^{3} a_{7}^{3} z^{9}+\cdots
$$

This is again an $E_{7}$ enhancement. The spectral cover equation is

$$
\left(a_{1}+a_{2} s+a_{3} s^{2}\right) a_{5} a_{7} s^{2}=0
$$

$\mathbf{S O}(12)$ enhancement. The $\mathrm{SO}(12)$ is obtained setting $b_{3}=0$. This has two solutions: either

$$
a_{5} a_{6}+a_{4} a_{7}=0 \Rightarrow\left\{a_{5} \rightarrow \lambda a_{4}, a_{7} \rightarrow-\lambda a_{6}\right\}
$$

or

$$
a_{1} \rightarrow \lambda a_{4} a_{6}
$$

Substituting the first one in $b_{k}\left(a_{i}\right)$ 's of (A.8) we get

$$
\left(\lambda a_{4} a_{7}+a_{5}\left(\lambda a_{6}-\left(a_{1}+\lambda a_{4} a_{6}\right) a_{7}\right)\right)^{2}-4 \lambda a_{1} a_{4} a_{5}^{2} a_{6} a_{7}^{2}=\lambda^{4} a_{4}^{2} a_{6}^{2}\left(a_{1}-\lambda a_{4} a_{6}\right)^{2}
$$

If both conditions implying $b_{3}=0$ are imposed, the latter is also zero, leading to $\mathrm{SO}(14)$ enhancement. This enhancement can also happen if instead of the second condition $a_{1} \rightarrow$ $\lambda a_{4} a_{6}$ we impose $a_{4}=0$ or $a_{6}=0$. We summarize all the above cases in table 24 . In the last two entries of this table the $\tilde{t}_{1,2}$ are incorporated into the spectral cover. 


\begin{tabular}{|c|c|c|c|c|c|c|c|c|}
\hline$a_{1}$ & $a_{2}$ & $a_{3}$ & $a_{4}$ & $a_{5}$ & $a_{6}$ & $a_{7}$ & $a_{8}$ & $a_{9}$ \\
$\eta-2 c_{1}-\chi$ & $\eta-c_{1}-\chi$ & $\eta-\chi$ & $-c_{1}+x_{5}$ & $x_{5}$ & $-c_{1}+x_{7}$ & $x_{7}$ & $-c_{1}+\chi_{9}$ & $\chi_{9}$ \\
\hline
\end{tabular}

Table 25. Homology classes for coefficients $a_{i}$ for the $\mathcal{Z}_{2}(\mathrm{SU}(5))$ case.

\section{B.1.2 $\mathrm{SU}(5)$ and spectral cover splitting for the $\mathcal{Z}_{2}$ case}

The case of $\mathcal{Z}_{2}$ monodromy corresponds to the following splitting of the spectral cover equation

$$
b_{0} \prod_{i}\left(s-t_{i}\right)=\left(a_{1}+a_{2} s+a_{3} s^{2}\right)\left(a_{4}+a_{5} s\right)\left(a_{6}+a_{7} s\right)\left(a_{8}+a_{9} s\right)=\sum_{k=0}^{5} b_{k} s^{5-k}
$$

Following [21] we derive the relations of $b_{k}\left(a_{i}\right)$ by equating coefficients of the same powers in $s$

$$
\begin{aligned}
& b_{0}=a_{3} a_{5} a_{7} a_{9} \\
& b_{1}=a_{3} a_{5} a_{7} a_{8}+a_{3} a_{5} a_{6} a_{9}+a_{3} a_{4} a_{7} a_{9}+a_{2} a_{5} a_{7} a_{9} \\
& b_{2}=a_{3} a_{5} a_{6} a_{8}+a_{3} a_{4} a_{7} a_{8}+a_{2} a_{5} a_{7} a_{8}+a_{3} a_{4} a_{6} a_{9}+a_{2} a_{5} a_{6} a_{9}+a_{2} a_{4} a_{7} a_{9}+a_{1} a_{5} a_{7} a_{9} \\
& b_{3}=a_{3} a_{4} a_{6} a_{8}+a_{2} a_{5} a_{6} a_{8}+a_{2} a_{4} a_{7} a_{8}+a_{1} a_{5} a_{7} a_{8}+a_{2} a_{4} a_{6} a_{9}+a_{1} a_{5} a_{6} a_{9}+a_{1} a_{4} a_{7} a_{9} \\
& b_{4}=a_{2} a_{4} a_{6} a_{8}+a_{1} a_{5} a_{6} a_{8}+a_{1} a_{4} a_{7} a_{8}+a_{1} a_{4} a_{6} a_{9} \\
& b_{5}=a_{1} a_{4} a_{6} a_{8}
\end{aligned}
$$

while to solve the constraint

$$
0=b_{1}=a_{3} a_{5} a_{7} a_{8}+a_{3} a_{4} a_{9} a_{7}+a_{2} a_{5} a_{7} a_{9}+a_{3} a_{5} a_{6} a_{9}
$$

we use the Ansatz

$$
a_{2}=-c\left(a_{5} a_{7} a_{8}+a_{4} a_{9} a_{7}+a_{5} a_{6} a_{9}\right), \quad a_{3}=c a_{5} a_{7} a_{9}
$$

Notice that setting $a_{8}=0, a_{9}=1$ this complies with our previous $\mathrm{SO}(10)$ Ansatz (B.2). The tenplets are found by studying the zeroth order of the above polynomial, which is

$$
b_{5}=t_{1} t_{2} t_{3} t_{4} t_{5}=a_{1} a_{4} a_{6} a_{8}
$$

These are designated as

$$
10_{t_{1}}^{(1)}, 10_{t_{3}}^{(2)}, 10_{t_{4}}^{(3)}, 10_{t_{5}}^{(4)}
$$

while their homologies are associated to those of $a_{1}, a_{4}, a_{6}, a_{8}$. To determine the properties of the fiveplets we need the corresponding spectral cover equation. This is a 10-degree polynomial

$$
\mathcal{P}_{10}(s) \propto \sum_{n=1}^{10} c_{n} s^{10-n}=b_{0} \prod_{i, j}\left(s-t_{i}-t_{j}\right), \quad i<j, \quad i, j=1, \ldots, 5
$$




\begin{tabular}{|c|c|c|c|c|c|}
\hline$a_{1}$ & $a_{2}$ & $a_{3}$ & $a_{4}$ & $a_{5}$ & $a_{6}$ \\
$\eta-2 c_{1}-\chi$ & $\eta-c_{1}-\chi$ & $\eta-\chi$ & $\chi-2 c_{1}$ & $\chi-c_{1}$ & $\chi$ \\
\hline
\end{tabular}

Table 26. Homology classes for coefficients $a_{i}$ for the $\mathcal{Z}_{2} \times \mathcal{Z}_{2}$ case.

Using (B.5) we can convert the coefficients $c_{n}=c_{n}\left(t_{j}\right)$ to functions of $c_{n}\left(b_{j}\right)$. In particular we are interested for the the value $\mathcal{P}_{10}(0)$ given by the coefficient $c_{10}$ which can be expressed in terms of $b_{k}$ according to

$$
c_{10}\left(b_{k}\right)=b_{3}^{2} b_{4}-b_{2} b_{3} b_{5}+b_{0} b_{5}^{2}=0
$$

Using the equations $b_{k}\left(a_{i}\right)$ and the Ansatz, we can split this equation into seven factors which correspond to the seven distinct fiveplets left after the $\mathcal{Z}_{2}$ monodromy action given in $(2.16)$ and in table 2.

\section{B.2 $\mathcal{Z}_{2} \times \mathcal{Z}_{2}$ spectral cover factorization}

In the case of $C_{2+2}$ factorization we write the polynomial $P_{16}(s)$ as follows

$$
P_{16}(s)=\left(a_{3} s^{2}+a_{2} s+a_{1}\right)\left(a_{6} s^{2}+a_{5} s+a_{4}\right)
$$

This splitting implies the $t_{1} \leftrightarrow t_{2}$ and $t_{3} \leftrightarrow t_{4}$ identifications. Comparing with the coefficients $b_{k}$ we have

$$
\begin{aligned}
& b_{4}=a_{1} a_{4} \\
& b_{3}=a_{2} a_{4}+a_{1} a_{5} \\
& b_{2}=a_{3} a_{4}+a_{2} a_{5}+a_{1} a_{6} \\
& b_{1}=a_{3} a_{5}+a_{2} a_{6} \\
& b_{0}=a_{3} a_{6}
\end{aligned}
$$

Starting from the equation $b_{1}=0$, we see that the acceptable Ansatz is

$$
a_{3}=\lambda a_{6}, \quad a_{2}=-\lambda a_{5}
$$

In this case the $b_{k}$ 's are given by

$$
\begin{aligned}
& b_{4}=a_{1} a_{4} \\
& b_{3}=\left(a_{1}-\lambda a_{4}\right) a_{5} \\
& b_{2}=\left(a_{1}+\lambda a_{4}\right) a_{6}-\lambda a_{5}^{2} \\
& b_{1}=0 \\
& b_{0}=\lambda a_{6}^{2}
\end{aligned}
$$

Repeating the same steps as in the $\mathcal{Z}_{2}$ case we determine the homologies of $a_{i}$ given in table 26 and the properties of the matter curves given in table 3 . Next we recall that the $E_{6}$ enhancement is obtained for $b_{4}=a_{1} a_{4}=0$ and this can be done by demanding either of $a_{1,4}$ to be zero. The case $a_{1}=0$ implies

$$
\mathcal{C}_{4}=s\left(a_{2}+a_{3} s\right)\left(a_{4}+a_{5} s+a_{6} s^{2}\right)
$$




\begin{tabular}{|c|c|c|}
\hline$E_{6}$ & $E_{7}$ & $\mathrm{U}(1)$ embedding $i=1,2, j=3,4$ \\
\hline$a_{1}=0$ & $a_{4}=0$ & $t_{i, j} \in E_{7} \times \mathrm{SU}(2)$ \\
$a_{1}=0$ & $a_{5}=0$ & $t_{i} \in E_{7}, \tilde{t}_{j} \in \mathrm{SU}(2)$ \\
$a_{4}=0$ & $a_{5}=0$ & $\tilde{t}_{i} \in \mathrm{SU}(2), t_{j} \in E_{7}$ \\
\hline
\end{tabular}

Table 27. The vanishing coefficients with the corresponding enhancements and the embedding of the $\mathrm{U}(1)$ 's involved in the monodromy. In the first case the monodromies are between a $\mathrm{U}(1) \in E_{7}$ and a $\mathrm{U}(1) \in \mathrm{SU}(2)$.

\begin{tabular}{|c|c|c|c|c|c|c|c|}
\hline$a_{1}$ & $a_{2}$ & $a_{3}$ & $a_{4}$ & $a_{5}$ & $a_{6}$ & $a_{7}$ & $a_{8}$ \\
$\eta-2 c_{1}-\chi-\psi$ & $\eta-c_{1}-\chi-\psi$ & $\eta-\chi-\psi$ & $\chi-2 c_{1}$ & $\chi-c_{1}$ & $\chi$ & $-c_{1}+\psi$ & $\psi$ \\
\hline
\end{tabular}

Table 28. Homology classes for coefficients $a_{i}$ for the $\mathcal{Z}_{2} \times \mathcal{Z}_{2}$ case for $\mathrm{SU}(5)$.

Thus for the particular choice $a_{1}=0$ at the first order enhancement to $E_{6}$ one $\mathcal{Z}_{2}$ monodromy is 'resolved' with one of the two U(1) incorporated into the $E_{6}$ symmetry. (The same happens for $a_{4}=0$.) The $E_{7}$ enhancement is reached when in addition we have $b_{3}=0$ and this happens either when $a_{1}=a_{4}=0$ or $a_{1}=a_{5}=0$ (or $a_{4}=0=a_{5}=0$ ). The cases are collected in table 27 .

\section{B.2.1 The SU(5) case}

In the $\mathcal{Z}_{2} \times \mathcal{Z}_{2}$ case, the spectral cover equation for $\mathrm{SU}(5)$ obtains the form

$$
\mathcal{C}_{10}(s)=\left(a_{3} s^{2}+a_{2} s+a_{1}\right)\left(a_{6} s^{2}+a_{5} s+a_{4}\right)\left(a_{7}+a_{8} s\right)
$$

Proceeding as in the $\mathrm{SO}(10)$ case, we identify the relations $b_{k}\left(a_{i}\right), k=1, \ldots 5$ by comparing coefficients of the same power in $s$.

$$
\begin{aligned}
& b_{0}=a_{3} a_{6} a_{8} \\
& b_{1}=a_{3} a_{6} a_{7}+a_{3} a_{5} a_{8}+a_{2} a_{6} a_{8} \\
& b_{2}=a_{3} a_{5} a_{7}+a_{2} a_{6} a_{7}+a_{3} a_{4} a_{8}+a_{2} a_{5} a_{8}+a_{1} a_{6} a_{8} \\
& b_{3}=a_{3} a_{4} a_{7}+a_{2} a_{5} a_{7}+a_{1} a_{6} a_{7}+a_{2} a_{4} a_{8}+a_{1} a_{5} a_{8} \\
& b_{4}=a_{2} a_{4} a_{7}+a_{1} a_{5} a_{7}+a_{1} a_{4} a_{8} \\
& b_{5}=a_{1} a_{4} a_{7}
\end{aligned}
$$

The constraint $b_{1}=0$ is solved by the Ansatz

$$
a_{3}=\lambda a_{6} a_{8}, \quad a_{2}=-\lambda\left(a_{6} a_{7}+a_{5} a_{8}\right)
$$

Notice that this reduces to SU(4) case (B.10) when $a_{7}=0, a_{8}=1$. Solving the equations analogously to the previous cases, we can easily determine the homology classes of $a_{i}$ given in table 28.

The $10 \in \mathrm{SU}(5)$ are obtained from the solutions of the equation

$$
b_{5}=0, \rightarrow a_{1} a_{4} a_{7}=0
$$


therefore they are associated to $a_{1}=0, a_{4}=0$ and $a_{7}=0$. The fiveplets are found by solving the corresponding equation

$$
b_{3}^{2} b_{4}-b_{2} b_{3} b_{5}+b_{0} b_{5}^{2}=0
$$

It is straightforward to determine the homology classes and other properties using the results of table 28. The results are summarized in 2.22 and in table 4 in the main body of the paper.

\section{B.3 $\mathcal{Z}_{3}$ factorization}

We write the spectral cover equation as follows

$$
\mathcal{C}_{4}=\left(a_{1}+a_{2} s+a_{3} s^{2}+a_{4} s^{3}\right)\left(a_{5}+s a_{6}\right)
$$

Comparing with the coefficients $b_{k}$, we get

$$
\begin{aligned}
& b_{4}=a_{1} a_{5} \\
& b_{3}=a_{2} a_{5}+a_{1} a_{6} \\
& b_{2}=a_{3} a_{5}+a_{2} a_{6} \\
& b_{1}=a_{4} a_{5}+a_{3} a_{6} \\
& b_{0}=a_{4} a_{6}
\end{aligned}
$$

Imposing the conditions $a_{5}=\lambda a_{6}, a_{3}=-\lambda a_{4}$ we get

$$
\begin{aligned}
& b_{4}=\lambda a_{1} a_{6} \\
& b_{3}=\left(a_{1}+\lambda a_{2}\right) a_{6} \\
& b_{2}=\left(a_{2}-\lambda^{2} a_{4}\right) a_{6} \\
& b_{1}=0 \\
& b_{0}=a_{4} a_{6}
\end{aligned}
$$

The $b_{4}=0$ enhancement to $E_{6}$ is obtained by $a_{1}=0$ while the condition $b_{3}=0$ associated to the $E_{7}$ enhancement is obtained by setting $a_{2}=0$. Notice however that in this Ansatz the condition $a_{6}=0$ eliminates all $b_{k}$ 's. A non-trivial solution is given by the Ansatz $a_{4}=\lambda a_{6}, a_{3}=-\lambda a_{4}$ which entails the following forms of $b_{k}$ 's

$$
\begin{aligned}
& b_{4}=a_{1} a_{5} \\
& b_{3}=a_{1} a_{6}+a_{2} a_{5} \\
& b_{2}=a_{2} a_{6}-\lambda a_{5}^{2} \\
& b_{1}=0 \\
& b_{0}=\lambda a_{6}^{2}
\end{aligned}
$$

It is straightforward to correlate the $E_{6}, E_{7}$ enhancements with the vanishing of the appropriate coefficients $a_{i}$.

The homology classes of $a_{i}$ are shown in table 29. The results are collected in table 5 . 


\begin{tabular}{|c|c|c|c|c|c|}
\hline$a_{1}$ & $a_{2}$ & $a_{3}$ & $a_{4}$ & $a_{5}$ & $a_{6}$ \\
$\eta-3 c_{1}-\chi$ & $\eta-2 c_{1}-\chi$ & $\eta-c_{1}-\chi$ & $\eta-\chi$ & $\chi-c_{1}$ & $\chi$ \\
\hline
\end{tabular}

Table 29. Homology classes for coefficients $a_{i}$ for the $\mathcal{Z}_{3}$ case in the $\mathrm{SO}(10)$ model.

\begin{tabular}{|c|c|c|c|c|c|c|c|}
\hline$a_{1}$ & $a_{2}$ & $a_{3}$ & $a_{4}$ & $a_{5}$ & $a_{6}$ & $a_{7}$ & $a_{8}$ \\
$\eta-3 c_{1}-\chi-\psi$ & $\eta-2 c_{1}-\chi-\psi$ & $\eta-c_{1}-\chi-\psi$ & $\eta-\chi-\psi$ & $\chi-c_{1}$ & $\chi$ & $\psi-c_{1}$ & $\psi$ \\
\hline
\end{tabular}

Table 30. SU(5): homology classes for coefficients $a_{i}$ for the $\mathcal{Z}_{3}$ case.

\section{B.3.1 SU(5) with $\mathcal{Z}_{3}$ monodromy}

In this case the relevant spectral cover polynomial is

$$
\sum_{k=0}^{5} b_{k} s^{5-k}=\left(a_{4} s^{3}+a_{3} s^{2}+a_{2} s+a_{1}\right)\left(a_{5}+s a_{6}\right)\left(a_{7}+s a_{8}\right)
$$

We can easily extract the equations determining the coefficients $b_{k}\left(a_{i}\right)$, by equating equal powers of $s$

$$
\begin{aligned}
& b_{0}=a_{4} a_{6} a_{8} \\
& b_{1}=a_{4} a_{6} a_{7}+a_{4} a_{5} a_{8}+a_{3} a_{6} a_{8} \\
& b_{2}=a_{4} a_{5} a_{7}+a_{3} a_{6} a_{7}+a_{3} a_{5} a_{8}+a_{2} a_{6} a_{8} \\
& b_{3}=a_{3} a_{5} a_{7}+a_{2} a_{6} a_{7}+a_{2} a_{5} a_{8}+a_{1} a_{6} a_{8} \\
& b_{4}=a_{2} a_{5} a_{7}+a_{1} a_{6} a_{7}+a_{1} a_{5} a_{8} \\
& b_{5}=a_{1} a_{5} a_{7}
\end{aligned}
$$

For each of the above equations, there is a corresponding one for the homologies

$\left[b_{k}\right]=\eta-k c_{1}=\left[a_{l}\right]+\left[a_{m}\right]+\left[a_{n}\right], \quad k=0,1, \ldots, 5, \quad k+l+m+n=18, \quad l, m, n \leq 8$ the latter being valid only for the combinations of indices appearing in (B.17). The homology classes of $a_{i}$ are shown in table 30. The condition

$$
0=b_{1}=a_{4} a_{6} a_{7}+a_{4} a_{5} a_{8}+a_{3} a_{6} a_{8}
$$

is solved using the Ansatz

$$
a_{3} \rightarrow-c\left(a_{6} a_{7}+a_{5} a_{8}\right), \quad a_{4} \rightarrow c a_{6} a_{8}
$$

which, again as expected, reduces to the corresponding SU(4) case (B.16) when $a_{7}=0$, $a_{8}=1$. Substitution of this conditions into the solution $b_{k}\left(a_{i}\right)$ gives

$$
\begin{aligned}
& b_{0}=c a_{6}^{2} a_{8}^{2} \\
& b_{1}=0 \\
& b_{2}=\left(a_{2}-c a_{5} a_{7}\right) a_{6} a_{8}-c\left(a_{6}^{2} a_{7}^{2}+a_{5}^{2} a_{8}^{2}\right) \\
& b_{3}=\left(a_{6} a_{7}+a_{5} a_{8}\right)\left(a_{2}-c a_{5} a_{7}\right)+a_{1} a_{6} a_{8} \\
& b_{4}=\left(a_{6} a_{7}+a_{5} a_{8}\right) a_{1}+a_{2} a_{5} a_{7} \\
& b_{5}=a_{1} a_{5} a_{7}
\end{aligned}
$$


The tenplets are determined by $b_{5}=a_{1} a_{5} a_{7}=0$. Under the above Ansatz, in the case of $\mathcal{Z}_{3}$ monodromy the equation (2.15) factorizes as in (2.24). These, together with the tenplets are given in table 7 .

Open Access. This article is distributed under the terms of the Creative Commons Attribution License which permits any use, distribution and reproduction in any medium, provided the original author(s) and source are credited.

\section{References}

[1] C. Vafa, Evidence for F-theory, Nucl. Phys. B 469 (1996) 403 [hep-th/9602022] [InSPIRE].

[2] R. Donagi and M. Wijnholt, Model building with F-theory, arXiv:0802.2969 [INSPIRE].

[3] C. Beasley, J.J. Heckman and C. Vafa, GUTs and exceptional branes in F-theory. I, JHEP 01 (2009) 058 [arXiv:0802.3391] [INSPIRE].

[4] R. Donagi and M. Wijnholt, Breaking GUT groups in F-theory, arXiv:0808.2223 [INSPIRE].

[5] C. Beasley, J.J. Heckman and C. Vafa, GUTs and exceptional branes in F-theory. II: Experimental predictions, JHEP 01 (2009) 059 [arXiv:0806.0102] [INSPIRE].

[6] R. Blumenhagen, T.W. Grimm, B. Jurke and T. Weigand, Global F-theory GUTs, Nucl. Phys. B 829 (2010) 325 [arXiv:0908.1784] [INSPIRE].

[7] R. Donagi and M. Wijnholt, Higgs bundles and UV completion in F-theory, arXiv:0904.1218 [INSPIRE].

[8] H. Hayashi, R. Tatar, Y. Toda, T. Watari and M. Yamazaki, New aspects of heterotic-F theory duality, Nucl. Phys. B 806 (2009) 224 [arXiv:0805.1057] [INSPIRE].

[9] A. Collinucci, New F-theory lifts, JHEP 08 (2009) 076 [arXiv:0812.0175] [INSPIRE].

[10] A. Collinucci, New F-theory lifts. II: Permutation orientifolds and enhanced singularities, JHEP 04 (2010) 076 [arXiv:0906.0003] [inSPIRE].

[11] R. Blumenhagen, T.W. Grimm, B. Jurke and T. Weigand, F-theory uplifts and GUTs, JHEP 09 (2009) 053 [arXiv:0906.0013] [INSPIRE].

[12] J.J. Heckman, A. Tavanfar and C. Vafa, The point of $E_{8}$ in F-theory GUTs, JHEP 08 (2010) 040 [arXiv:0906.0581] [INSPIRE].

[13] H. Hayashi, T. Kawano, R. Tatar and T. Watari, Codimension-3 singularities and Yukawa couplings in F-theory, Nucl. Phys. B 823 (2009) 47 [arXiv:0901.4941] [InSPIRE].

[14] J. Marsano, N. Saulina and S. Schäfer-Nameki, Monodromies, fluxes and compact three-generation F-theory GUTs, JHEP 08 (2009) 046 [arXiv:0906.4672] [INSPIRE].

[15] J.J. Heckman and C. Vafa, Flavor hierarchy from F-theory, Nucl. Phys. B 837 (2010) 137 [arXiv: 0811.2417] [INSPIRE].

[16] A. Font and L.E. Ibáñez, Yukawa structure from U(1) fluxes in F-theory grand unification, JHEP 02 (2009) 016 [arXiv:0811.2157] [INSPIRE].

[17] J. Marsano, N. Saulina and S. Schäfer-Nameki, Compact F-theory GUTs with U(1) PQ , JHEP 04 (2010) 095 [arXiv: 0912.0272] [INSPIRE].

[18] J. Marsano, N. Saulina and S. Schäfer-Nameki, F-theory compactifications for supersymmetric GUTs, JHEP 08 (2009) 030 [arXiv:0904.3932] [INSPIRE]. 
[19] E. Dudas and E. Palti, Froggatt-Nielsen models from $E_{8}$ in F-theory GUTs, JHEP 01 (2010) 127 [arXiv:0912.0853] [INSPIRE].

[20] S.F. King, G.K. Leontaris and G.G. Ross, Family symmetries in F-theory GUTs, Nucl. Phys. B 838 (2010) 119 [arXiv: 1005.1025] [InSPIRE].

[21] E. Dudas and E. Palti, On hypercharge flux and exotics in F-theory GUTs, JHEP 09 (2010) 013 [arXiv:1007.1297] [INSPIRE].

[22] G.K. Leontaris and G.G. Ross, Yukawa couplings and fermion mass structure in F-theory GUTs, JHEP 02 (2011) 108 [arXiv: 1009.6000] [INSPIRE].

[23] C. Lüdeling, H.P. Nilles and C.C. Stephan, The potential fate of local model building, Phys. Rev. D 83 (2011) 086008 [arXiv:1101.3346] [INSPIRE].

[24] J.C. Callaghan, S.F. King, G.K. Leontaris and G.G. Ross, Towards a realistic F-theory GUT, JHEP 04 (2012) 094 [arXiv: 1109.1399] [INSPIRE].

[25] S. Cecotti, M.C.N. Cheng, J.J. Heckman and C. Vafa, Yukawa couplings in F-theory and non-commutative geometry, arXiv:0910.0477 [INSPIRE].

[26] S. Cecotti, C. Cordova, J.J. Heckman and C. Vafa, T-branes and monodromy, JHEP 07 (2011) 030 [arXiv:1010.5780] [INSPIRE].

[27] P.G. Camara, E. Dudas and E. Palti, Massive wavefunctions, proton decay and FCNCs in local F-theory GUTs, JHEP 12 (2011) 112 [arXiv:1110.2206] [INSPIRE].

[28] C.-M. Chen, J. Knapp, M. Kreuzer and C. Mayrhofer, Global SO(10) F-theory GUTs, JHEP 10 (2010) 057 [arXiv: 1005.5735] [INSPIRE].

[29] T.W. Grimm, S. Krause and T. Weigand, F-theory GUT vacua on compact Calabi-Yau fourfolds, JHEP 07 (2010) 037 [arXiv: 0912.3524] [INSPIRE].

[30] J. Jiang, T. Li, D.V. Nanopoulos and D. Xie, F-SU(5), arXiv:0811.2807 [InSPIRE].

[31] C.-M. Chen and Y.-C. Chung, A note on local GUT models in F-theory, Nucl. Phys. B 824 (2010) 273 [arXiv:0903.3009] [InSPIRE].

[32] J. Marsano, N. Saulina and S. Schäfer-Nameki, A note on G-fluxes for F-theory model building, JHEP 11 (2010) 088 [arXiv:1006.0483] [INSPIRE].

[33] L. Aparicio, A. Font, L.E. Ibáñez and F. Marchesano, Flux and instanton effects in local F-theory models and hierarchical fermion masses, JHEP 08 (2011) 152 [arXiv:1104.2609] [INSPIRE].

[34] E. Palti, Wavefunctions and the point of $E_{8}$ in F-theory, JHEP 07 (2012) 065 [arXiv: 1203.4490] [INSPIRE].

[35] D. Cremades, L.E. Ibáñez and F. Marchesano, Yukawa couplings in intersecting D-brane models, JHEP 07 (2003) 038 [hep-th/0302105] [INSPIRE].

[36] I. Antoniadis, A. Kumar and B. Panda, Fermion wavefunctions in magnetized branes: theta identities and Yukawa couplings, Nucl. Phys. B 823 (2009) 116 [arXiv:0904.0910] [INSPIRE].

[37] P.G. Camara and F. Marchesano, Open string wavefunctions in flux compactifications, JHEP 10 (2009) 017 [arXiv:0906.3033] [INSPIRE].

[38] F. Marchesano, P. McGuirk and G. Shiu, Chiral matter wavefunctions in warped compactifications, JHEP 05 (2011) 090 [arXiv: 1012.2759] [INSPIRE]. 
[39] S. Krippendorf, M.J. Dolan, A. Maharana and F. Quevedo, D-branes at toric singularities: model building, Yukawa couplings and flavour physics, JHEP 06 (2010) 092 [arXiv: 1002.1790] [INSPIRE].

[40] R. Donagi and M. Wijnholt, Gluing branes. I, arXiv:1104.2610 [INSPIRE].

[41] R. Donagi and M. Wijnholt, Gluing branes. II: Flavour physics and string duality, arXiv:1112.4854 [INSPIRE].

[42] J. Tate, Algorithm for determining the type of a singular fiber in an elliptic pencil, in Modular functions of one variable IV, Springer-Verlag, Berlin Germany (1975), Lect. Notes Math. 476 (1975) 33.

[43] C.D. Froggatt and H.B. Nielsen, Hierarchy of quark masses, Cabibbo angles and CP-violation, Nucl. Phys. B 147 (1979) 277 [INSPIRE].

[44] J. Marsano, Hypercharge flux, exotics and anomaly cancellation in F-theory GUTs, Phys. Rev. Lett. 106 (2011) 081601 [arXiv:1011.2212] [InSPIRE].

[45] H. Hayashi, T. Kawano, Y. Tsuchiya and T. Watari, Flavor structure in F-theory compactifications, JHEP 08 (2010) 036 [arXiv:0910.2762] [INSPIRE].

[46] R. Blumenhagen, Gauge coupling unification in F-theory grand unified theories, Phys. Rev. Lett. 102 (2009) 071601 [arXiv:0812.0248] [INSPIRE].

[47] G.K. Leontaris and N.D. Tracas, Gauge coupling flux thresholds, exotic matter and the unification scale in F-SU(5) GUT, Eur. Phys. J. C 67 (2010) 489 [arXiv:0912.1557] [INSPIRE].

[48] J.J. Heckman, C. Vafa and B. Wecht, The conformal sector of F-theory GUTs, JHEP 07 (2011) 075 [arXiv:1103.3287] [INSPIRE].

[49] G.K. Leontaris and N.D. Vlachos, On the GUT scale of F-theory SU(5), Phys. Lett. B 704 (2011) 620 [arXiv: 1105.1858] [INSPIRE].

[50] M.J. Dolan, J. Marsano and S. Schäfer-Nameki, Unification and phenomenology of F-theory GUTs with $\mathrm{U}(1)_{\mathrm{PQ}}$, JHEP 12 (2011) 032 [arXiv: 1109.4958] [INSPIRE].

[51] R. Davies, Dirac gauginos and unification in F-theory, arXiv:1205.1942 [INSPIRE].

[52] I. Antoniadis, E. Kiritsis, J. Rizos and T.N. Tomaras, D-branes and the standard model, Nucl. Phys. B 660 (2003) 81 [hep-th/0210263] [INSPIRE].

[53] V. Bouchard, J.J. Heckman, J. Seo and C. Vafa, F-theory and neutrinos: Kaluza-Klein dilution of flavor hierarchy, JHEP 01 (2010) 061 [arXiv:0904.1419] [INSPIRE].

[54] H.M. Lee et al., Discrete $R$ symmetries for the MSSM and its singlet extensions, Nucl. Phys. B 850 (2011) 1 [arXiv:1102.3595] [INSPIRE].

[55] L.E. Ibáñez, A.N. Schellekens and A.M. Uranga, Discrete gauge symmetries in discrete MSSM-like orientifolds, arXiv:1205.5364 [INSPIRE].

[56] M. Bershadsky et al., Geometric singularities and enhanced gauge symmetries, Nucl. Phys. B 481 (1996) 215 [hep-th/9605200] [INSPIRE]. 